\title{
The stellar content of low redshift BL Lacertae host galaxies from multicolour imaging ${ }^{\star}$
}

\author{
T. Hyvönen ${ }^{1}$, J. K. Kotilainen ${ }^{1}$, R. Falomo ${ }^{2}$, E. Örndahl ${ }^{1}$, and T. Pursimo ${ }^{3}$ \\ 1 Tuorla Observatory, University of Turku, Väisäläntie 20, 21500 Piikkiö, Finland \\ e-mail: [totahy; jarkot]@utu.fi \\ 2 INAF - Osservatorio Astronomico di Padova, Vicolo dell'Osservatorio 5, 35122 Padova, Italy \\ e-mail: renato.falomo@oapd.inaf.it \\ 3 Nordic Optical Telescope, Apartado 474, 38700 Santa Cruz de La Palma, Santa Cruz de Tenerife, Spain \\ e-mail: tpursimo@not.iac.es
}

Received 2 July 2007 / Accepted 10 September 2007

\begin{abstract}
Context. We present $B$-band imaging of 18 low redshift $(z \leq 0.3)$ BL Lac objects for which their host galaxies were previously resolved in the $R$-band and the near-infrared $H$-band. For a subset of the objects, $U$ - and $V$-band imaging also is presented.

Aims. These multiwavelength data are used to investigate the blue-red-near-infrared colours and the colour gradients of the host galaxies of BL Lacs in comparison with other elliptical galaxies with and without nuclear activity.

Methods. For all the BL Lacs observed in the $B$-and $V$-bands, and all objects at $z<0.15$ in the $U$-band, the host galaxy is clearly resolved. In all cases galaxies are well represented by an elliptical model, with average absolute magnitude $M_{B}=-21.6 \pm 0.7$ and average scale length $R_{\mathrm{e}}=7.6 \pm 3.2 \mathrm{kpc}$. BL Lac host galaxies are therefore luminous (massive) elliptical galaxies, in agreement with previous studies in other bands.

Results. The best-fit $B$-band Kormendy relation of $\left(\mu_{\mathrm{e}}=3.3 \log R_{\mathrm{e}}(\mathrm{kpc})+18.4 \mathrm{mag} \operatorname{arcsec}^{-2}\right)$ is in reasonable agreement with that obtained for normal ellipticals and radio galaxies. This structural and dynamical similarity indicates that all massive elliptical galaxies can experience nuclear activity without significant perturbation of their global structure. The distributions of the integrated blue/nearinfrared colour (with average $B-H=3.5 \pm 0.5$ ) and colour gradient (with average $\Delta(B-R) / \Delta(\log r)=-0.14 \pm 0.75)$ of the BL Lac hosts are much wider than those for normal ellipticals, and most BL Lac objects have bluer hosts and/or steeper colour gradients than those in normal ellipticals.

Conclusions. The blue colours are likely caused by a young stellar population component, and indicates a link between star formation caused by an interaction/merging event and the onset of the nuclear activity. This result is corroborated by stellar population modelling, indicating a presence of young/intermediate age populations in the majority of the sample, in agreement with low redshift quasar hosts. The lack of strong signs of interaction may require a significant time delay between the event with associated star formation episodes and the start of the nuclear activity.
\end{abstract}

Key words. galaxies: active - galaxies: BL Lacertae objects: general - galaxies: interactions - galaxies: nuclei - galaxies: photometry - galaxies: structure

\section{Introduction}

BL Lac objects are an extreme class of active galactic nuclei (AGN) characterized by luminous, variable and polarized continuum emission across the electromagnetic spectrum and strong core-dominated radio emission with apparent superluminal motion (e.g., Kollgaard et al. 1992). These properties suggest that they are strongly beamed objects dominated by synchrotron emission from a relativistic jet aligned close to the line of sight (Blandford \& Rees 1978). BL Lac objects have many similarities to flat spectrum radio quasars (FSRQ) and they are often grouped together as blazars. According to the unified model of radio-loud AGN (Urry \& Padovani 1995), the parent population of BL Lac objects and FSRQs are low luminosity core-dominated FR I radio galaxies (RG), and high luminosity lobe-dominated FR II RGs, respectively. Consequently, their orientation independent properties, such as host galaxies and environments, should be identical to those of their parent populations.

* Figures 9 and 10 are only available in electronic form at http://www . aanda.org
A number of optical (e.g., Falomo \& Kotilainen 1999; Urry et al. 2000; Nilsson et al. 2003; Heidt et al. 2004) and nearinfrared (NIR) (e.g., Kotilainen et al. 1998; Scarpa et al. 2000; Cheung et al. 2003; Kotilainen \& Falomo 2004; Kotilainen et al. 2005) imaging studies have shown that virtually all nearby $(z<$ $0.5) \mathrm{BL}$ Lac objects are hosted in large and luminous elliptical galaxies, with average $M_{R} \sim-24.0$ and average scale length $R_{\mathrm{e}} \sim 10 \mathrm{kpc}$, similarly to both FR I and FR II RGs (Govoni et al. 2000). BL Lac hosts are much brighter than $L^{*}$ galaxies (the characteristic luminosity of the Schechter luminosity function: $M_{R}^{*}=-21.2$, Gardner et al. 1997; Nakamura et al. 2003). While the morphology, such as jets and close companions, of some of the hosts indicates a recent interaction, the large majority of them is indistinguishable from inactive massive ellipticals at similar redshift (Scarpa et al. 2000).

Until recently, however, imaging of BL Lac host galaxies was obtained in one band only (usually $R$-band). Therefore, little colour information exists for them, especially in the blue domain of the spectrum, where only a few objects have been studied in the $B$-band and even fewer in the $U$-band. 
Kotilainen \& Falomo (2004) observed a sample of 23 low redshift $(z<0.3)$ BL Lac objects in the $H$-band and combined with previous $H$-band imaging (Kotilainen et al. 1998; Scarpa et al. 2000; Cheung et al. 2003) and optical $R$-band data from the literature (Falomo \& Kotilainen 1999; Urry et al. 2000), investigated the integrated $R-H$ colours and colour gradients of a sample of 41 BL Lac host galaxies. They found that BL Lac host galaxies appear to be systematically bluer than inactive ellipticals and have a much wider distribution of host galaxy $R-H$ colour (average $R-H=2.2 \pm 0.4$ ) and steeper colour gradient (average $\Delta(R-H) / \Delta(\log r)=-0.38 \pm 0.60)$ than those for inactive elliptical galaxies with a dominant old stellar population (Peletier et al. 1990). Similar behaviour has been found for the colours and colour gradients in low redshift RGs (Govoni et al. 2000) and in low and intermediate redshift AGN and quasars with elliptical hosts (Schade et al. 2000; Örndahl et al. 2003; Jahnke et al. 2004; Sanchez et al. 2004). The blue colours are most likely caused by a young stellar population, indicating a recent star formation (SF) episode, possibly triggered by interaction or merging between galaxies. The wide distribution of colours probably reflects an object-to-object difference in the age of the most recent SF episode. Note that there is also spectroscopic evidence for young/intermediate age populations in AGN hosts (e.g., Nolan et al. 2001; Raimann et al. 2005).

In this study, we present multicolour optical imaging of a subsample of 18 BL Lac objects from the large, homogeneous sample of 41 sources for which high resolution $R$ - and $H$-band imaging exists (Kotilainen et al. 1998; Scarpa et al. 2000; Cheung et al. 2003; Kotilainen \& Falomo 2004). Most of the observed objects have bluer $R-H$ host colour than inactive ellipticals and blue band observations are paramount to assess whether their blue colours are caused by a young stellar population. All the $18 \mathrm{BL}$ Lac objects were observed in the $B$ band, while for a subsample, we also obtained $U$ - and $V$-band imaging. The $U$-band observations were mainly restricted to the most nearby targets in the sample. Combining the deep high spatial resolution $U-, B$ - and $V$-band imaging with the existing $R$ and $H$-band data, we are able to derive the colours and colour gradients over an extended wavelength range, where the $H$-band is sensitive to the old stellar population that dominates the host galaxy mass, while the blue part of the spectrum traces the contribution from young stellar populations to the excess blue light in the host galaxies. We also derive structural properties, such as morphology and effective radius, as well as the absolute magnitude of the host galaxies in each observed band. The obtained $U B V R H$ broad band colours are used in conjunction with stellar synthesis population models to estimate the ages of the most recent $\mathrm{SF}$ episode in the host galaxies.

In Sect. 2, we describe the sample, observations, data reduction, and methods of analysis. In Sect. 3, we present the results and discussion concerning the properties of the host galaxies. A summary and conclusions are given in Sect. 4. Throughout this paper, $H_{0}=70 \mathrm{~km} \mathrm{~s}^{-1} \mathrm{Mpc}^{-1}, \Omega_{\mathrm{m}}=0.3$ and $\Omega_{\Lambda}=0.7$ cosmology is used.

\section{Observations, data reduction and analysis}

The observations were carried out during several observing runs with different telescopes, with most of the observations done at the $2.5 \mathrm{~m}$ Nordic Optical Telescope (NOT) with the ALFOSC instrument using Bessel $U, B$ and $V$ broad band filters. Nine targets were observed only in the $B$-band, five in the $U B V$ bands and four in the $U B$-bands. One target (MRK 421) was additionally observed in the $H$-band, to complement the sample
Table 1. Properties of the telescopes and instruments ${ }^{a}$.

\begin{tabular}{llrl}
\hline \hline Band & Telescope+instrument & $N_{\text {obj }}$ & $\begin{array}{l}\text { Scale } \\
\operatorname{arcsec} \mathrm{px}^{-1} \\
(1)\end{array}$ \\
\hline$U, B, V$ & (2) & $(3)$ & $(4)$ \\
$U, B$ & NOT/ALFOSC & 26 & 0.190 \\
$H$ & NOT/NOTCA & 3 & 0.217 \\
$U, B$ & ESO NTT/EMMI & 1 & 0.235 \\
\hline
\end{tabular}

${ }^{a}$ Column (1) gives the observed band; (2) the telescope and instrument used; (3) the number of observed targets, and (4) the spatial scale of the instrument.

presented in Kotilainen \& Falomo (2004). The B-band image of 3C 371, published in Nilsson et al. (1997), was kindly provided by $\mathrm{K}$. Nilsson. A summary of the telescopes and instruments used is given in Table 1. Seeing during the observations varied between 0.9 and 2.3 arcsec $F W H M$ (average $1.5 \pm 0.5$ arcsec and median 1.3 arcsec). For each target, several integrations (typically of $600 \mathrm{~s}$ duration) were obtained. The journal of the observations and properties of the sample are given in Table 2.

Data reduction was performed in a standard fashion using IRAF $^{1}$. This consisted of bias subtraction, flat field division and cosmic ray rejection. For each night, bias and flat field images were made from several median combined bias frames and twilight flat field exposures and cosmic rays were rejected using IRAF procedures. Finally, individual images were aligned and combined to form the final image of the target. The observations were mostly done in photometric conditions (see Table 2) and several photometric standard stars from Landolt (1992) were observed during each night. However, some targets were observed during non-photometric nights and for these objects additional short exposures were subsequently obtained in photometric conditions to calibrate these frames using reference stars in the field. $K$-correction from Poggianti (1997) was applied to the host galaxy magnitudes, but not to the nuclear magnitudes, since the nuclear component can be assumed to have a powerlaw spectrum $\left(f_{v} \propto v^{-\alpha}\right)$ with $\alpha \sim-1$. Absolute magnitudes were also corrected for interstellar extinction calculated for each band from the $R$-band extinction coefficient from Urry et al. (2000).

To derive the properties of the host galaxies, azimuthally averaged 1D radial luminosity profiles were extracted for each BL Lac object and for a number of field stars. Any obvious extra features, such as nearby companions and/or foreground stars, were masked out from the image to avoid contamination of the radial luminosity profile. To obtain an accurate model for the nuclear region of the targets, it is important to have a well defined point spread function (PSF). In most cases there were a number of suitable stars in the relatively large field of view surrounding the objects to form a reliable PSF. The core and the wing of the PSF were derived from a faint and a bright star in the frame, respectively, and they were combined to form the final PSF model. This final PSF was compared with the profiles of individual stars in the frame to assure that the procedure resulted in a good and stable representation of the true PSF. A representative case of this PSF comparison is shown in Fig. 1, where the individual stellar profiles are in good agreement with the adopted PSF model well into the domain where the host galaxy

1 IRAF is distributed by the National Optical Astronomy Observatories, which are operated by the Association of Universities for Research in Astronomy, Inc., under cooperative agreement with the National Science Foundation. 
Table 2. The sample and the journal of observations ${ }^{a}$.

\begin{tabular}{|c|c|c|c|c|c|c|c|c|}
\hline $\begin{array}{l}\text { Name } \\
\text { (1) }\end{array}$ & (2) & (3) & $\begin{array}{l}M_{B} \\
\text { (4) }\end{array}$ & $\begin{array}{l}\text { Filter } \\
\text { (s) } \\
\text { (5) }\end{array}$ & $\begin{array}{r}T_{\exp } \\
(6)\end{array}$ & $\begin{array}{l}\text { Date } \\
(\operatorname{arcsec}) \\
(7)\end{array}$ & $\begin{array}{l}\text { FWHM } \\
\text { (8) }\end{array}$ & $\begin{array}{l}\text { Photometric? } \\
\text { (9) }\end{array}$ \\
\hline \multirow[t]{3}{*}{ 1ES 0229+200 } & 0.139 & 18.0 & -21.7 & $U$ & 2700 & $12 / 11 / 2004$ & 1.7 & $\mathrm{Y}$ \\
\hline & & & & $B$ & 900 & $12 / 11 / 2004$ & 1.9 & $\mathrm{Y}$ \\
\hline & & & & V & 1800 & $12 / 11 / 2004$ & 2.0 & $\mathrm{Y}$ \\
\hline PKS 0521-365 & 0.055 & 14.6 & -22.3 & $B$ & 480 & $14 / 03 / 2005$ & 1.4 & Y \\
\hline PKS 0548-322 & 0.069 & 15.5 & -22.0 & $B$ & 900 & $15 / 03 / 2005$ & 1.3 & $\mathrm{Y}$ \\
\hline \multirow[t]{3}{*}{$1 \mathrm{H} 0706+591$} & 0.125 & 19.5 & -21.0 & $U$ & 1600 & $27 / 01 / 2004$ & 1.2 & $\mathrm{Y}$ \\
\hline & & & & $B$ & 1500 & $27 / 01 / 2004$ & 0.9 & $\mathrm{Y}$ \\
\hline & & & & $V$ & 600 & $27 / 01 / 2004$ & 1.2 & $\mathrm{Y}$ \\
\hline \multirow[t]{3}{*}{ MRK 421} & 0.031 & 13.8 & -22.9 & $U$ & 900 & $08 / 03 / 2005$ & 0.9 & $\mathrm{~N}$ \\
\hline & & & & $B$ & 300 & $08 / 03 / 2005$ & 0.9 & $\mathrm{~N}$ \\
\hline & & & & $H$ & 150 & $25 / 03 / 2005$ & 1.8 & Y \\
\hline \multirow{2}{*}{ MRK 180} & 0.045 & 15.0 & -22.1 & $U$ & 900 & $06 / 05 / 2002$ & 1.2 & Y \\
\hline & & & & $B$ & 1600 & $10 / 03 / 2003$ & 1.2 & Y \\
\hline 1ES $1212+078$ & 0.136 & 16.1 & -23.6 & $B$ & 1800 & 08/03/2005 & 0.9 & $\mathrm{~N}$ \\
\hline MS $1229.2+6430$ & 0.164 & 16.9 & -21.7 & $B$ & 1800 & $10 / 03 / 2004$ & 2.1 & $\mathrm{Y}$ \\
\hline 1ES $1255+244$ & 0.140 & 15.4 & -24.3 & $B$ & 1800 & 09/03/2005 & 1.9 & $\mathrm{Y}$ \\
\hline \multirow{2}{*}{ PG 1418+546 } & 0.152 & 15.7 & -23.7 & $U$ & 900 & $07 / 05 / 2002$ & 2.1 & Y \\
\hline & & & & $B$ & 700 & $07 / 05 / 2002$ & 1.3 & Y \\
\hline 1ES $1426+428$ & 0.129 & 16.5 & -22.5 & $B$ & 1600 & $10 / 03 / 2003$ & 1.8 & $\mathrm{Y}$ \\
\hline 1ES $1440+122$ & 0.162 & 17.0 & -22.9 & $B$ & 1800 & $08 / 03 / 2005$ & 0.9 & $\mathrm{~N}$ \\
\hline \multirow[t]{2}{*}{ AP LIBRAE } & 0.049 & 14.8 & -21.7 & $U$ & 700 & $07 / 05 / 2002$ & 2.0 & $\mathrm{Y}$ \\
\hline & & & & $B$ & 900 & $15 / 03 / 2005$ & 1.1 & $\mathrm{Y}$ \\
\hline MS $1552.1+2020$ & 0.273 & 17.7 & -23.5 & $B$ & 1200 & 09/03/2005 & 1.9 & $\mathrm{Y}$ \\
\hline \multirow[t]{3}{*}{ MRK 501} & 0.034 & 13.8 & -22.4 & $U$ & 600 & $07 / 05 / 2002$ & 1.2 & $\mathrm{Y}$ \\
\hline & & & & $B$ & 240 & $08 / 03 / 2005$ & 1.1 & $\mathrm{~N}$ \\
\hline & & & & $V$ & 180 & $08 / 03 / 2005$ & 1.2 & $\mathrm{~N}$ \\
\hline \multirow[t]{3}{*}{ I Zw 187} & 0.055 & 16.4 & -21.1 & $U$ & 500 & $07 / 05 / 2002$ & 2.0 & Y \\
\hline & & & & $B$ & 900 & $25 / 09 / 2003$ & 1.1 & $\mathrm{Y}$ \\
\hline & & & & $V$ & 300 & $08 / 03 / 2005$ & 1.3 & $\mathrm{~N}$ \\
\hline \multirow[t]{3}{*}{ 3C 371} & 0.051 & 14.2 & -22.6 & $U$ & 1200 & $01 / 10 / 2000$ & 2.1 & Y \\
\hline & & & & $B$ & 3600 & 02/05/1997 & 0.8 & $\mathrm{Y}$ \\
\hline & & & & $V$ & 480 & $08 / 03 / 2005$ & 1.3 & $\mathrm{~N}$ \\
\hline BL LACERTAE & 0.069 & 14.7 & -22.4 & $B$ & 720 & $01 / 10 / 2000$ & 2.3 & $\mathrm{Y}$ \\
\hline
\end{tabular}

${ }^{a}$ Column (1) gives the name of the BL Lac object; (2) the redshift; (3) the $V$-band apparent magnitude; (4) the $B$-band absolute magnitude; (5) the filter used; (6) the total integration time; (7) the date of the observation; (8) the seeing FWHM and (9) photometric (Y) or non-photometric (N) conditions.

becomes dominant over the PSF. The only exception was the field of MRK 421 where there are no suitable stars available. In this case, the PSF was estimated using standard stars observed during the same night with similar seeing conditions.

The luminosity profiles were decomposed into a point source (represented by the PSF) and elliptical galaxy components by an iterative least-squares fit to the observed profile. There are three free parameters in the fit: the PSF normalization, the host galaxy normalization and the effective radius of the host galaxy. The data were fit using the $r^{1 / 4}$ de Vaucouleurs law for elliptical galaxies to represent the host galaxy. The host galaxy was considered to be resolved if the PSF + host galaxy fit resulted in a considerably lower $\chi^{2}$ value than the PSF fit only. The uncertainty in the derived host galaxy magnitudes was estimated to be $\sim 0.2$ mag.

\section{Results}

From the images, 1D azimuthally averaged radial luminosity profiles of the BL Lac objects were extracted in the $U-, B$ - and $V$-band. The $U$-band profiles together with the best-fit model overlaid are presented in Fig. 8. The $B$ - and $V$-band profiles are available in electronic form.
We were able to clearly resolve the host galaxy in all objects in the $B$-and $V$-bands, and in $8 / 9$ objects in the $U$-band. The only target that remained unresolved in the $U$-band, PG $1418+546$, is one of the most distant objects in the sample. The results of the modelling are summarized in Table 3.

\subsection{Luminosities and sizes of the host galaxies}

Figure 2 shows the distribution of the $B$-band absolute magnitudes of the BL Lac host galaxies (this work), low redshift radioloud (RLQ) and radio-quiet (RQQ) quasar hosts from Jahnke et al. (2004), low redshift RGs from Govoni et al. (2000) and inactive elliptical galaxies from Peletier et al. (1990), Colbert et al. (2001) and Bower et al. (1992). The average $B$-band absolute magnitudes of the BL Lac objects, quasar and RG host galaxies in these samples are presented in Table 4, where all absolute quantities were transformed into the cosmology adopted here.

Since the selection criteria of the various samples are somewhat non-homogeneous, there is a possibility of selection effects. They are likely to affect absolute magnitudes but since the main focus of this study is the colour properties of BL Lac hosts as compared with other ellipticals, our main results are 

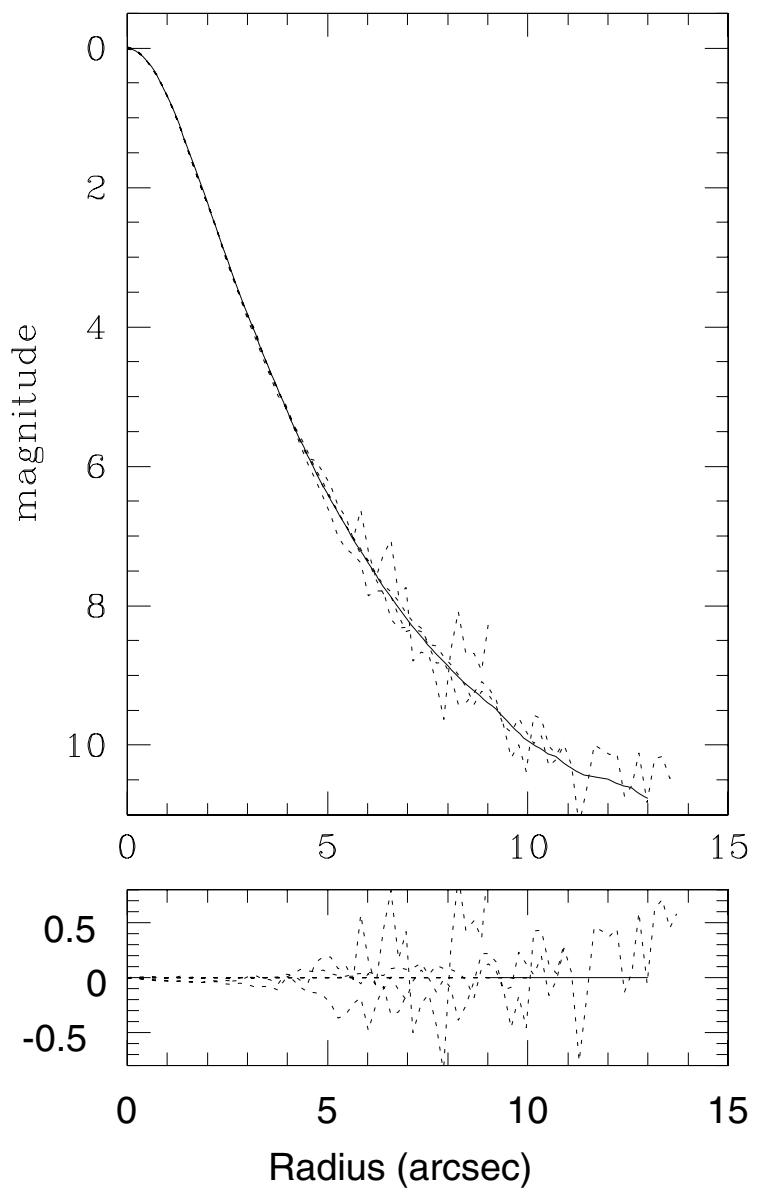

Fig. 1. Upper panel: comparison of the PSF profile extracted from field stars (the solid line) with the profiles of individual stars (dotted lines) in the field of BL Lac object. Lower panel: the difference between the profiles of individual stars and the PSF model.

only marginally affected by selection effects. Furthermore, our BL Lac sample is well matched to RGs and quasars in redshift (see Table 4) to minimize any bias introduced by possible evolutionary effects.

The average $B$-band absolute magnitude of the $18 \mathrm{BL}$ Lac hosts is $M_{B}=-21.6 \pm 0.7$, i.e. $\sim 1$ mag brighter than for low redshift quasar hosts (average $M_{B}=-20.9 \pm 0.4$ Jahnke et al. 2004). All the BL Lac hosts are in the luminosity range between $M_{B}^{*}+0.5$ and $M_{B}^{*}+2.5$, where $M_{B}^{*}=-20.8$ (Gardner et al. 1997; Nakamura et al. 2003) is the characteristic luminosity of the Schechter luminosity function for elliptical galaxies. The average luminosity of the BL Lac hosts is in agreement with inactive isolated early-type galaxies $\left(M_{B}=-21.5 \pm 0.7\right.$; Colbert et al. 2001) but is $\sim 1$ mag brighter than ellipticals observed by Peletier et al. (1990) $\left(M_{B}=-20.9 \pm 1.6\right)$. The distribution of inactive ellipticals has a tail toward fainter magnitudes that is not present in the BL Lac sample.

According to the unified model of AGN (Urry \& Padovani 1995), BL Lac objects are physically similar to FR I RGs but viewed from a different orientation. The classification of BL Lac objects with FR Is is based on their radio morphology, showing that BL Lac objects have similar extended radio properties to FR Is (e.g., Antonucci \& Ulvestad 1985), however, there are some indications that the radio properties of at least some BL Lac objects are similar to FR IIs rather than FR Is (e.g., Kollgaard et al. 1992; Stanghellini et al. 1997). There is also evidence that some BL Lac objects share the luminosity properties of FR IIs (Cassaro et al. 1999). The average B-band luminosity of low redshift FR I and FR II RGs is $M_{\text {FRI }}=-22.1 \pm 0.6$ and $M_{\text {FRII }}=-21.9 \pm 0.6$, respectively, obtained by Govoni et al. (2000). BL Lac hosts appear, therefore, to be slightly fainter than both FR Is and FR IIs, but based on Kolmogorov-Smirnov statistics, the luminosity distributions for the BL Lac hosts and the combined sample of RGs are indistinguishable. Our result suggests that both FR I and FR II RGs can be considered as a parent population of BL Lac objects, consistent with the result obtained by Falomo \& Kotilainen (1999).

The average effective radius of the BL Lac host galaxies in the $B$-band is $R_{\mathrm{e}}=7.6 \pm 3.2 \mathrm{kpc}$, consistent with results from previous studies (e.g., Nilsson et al. 2003; Falomo \& Kotilainen 1999) that BL Lac objects are hosted in large elliptical galaxies. BL Lac hosts are of similar size in the $B$-band to quasar host galaxies $\left(R_{\mathrm{e}}=6.0 \pm 2.7 \mathrm{kpc}\right.$; Jahnke et al. 2004) but they are slightly smaller than RGs in the $R$-band $\left(R_{\mathrm{e}}=13.6 \pm 8.7 \mathrm{kpc}\right)$; Govoni et al. 2000). Note, however, that the latter comparison may be biased by a luminosity-dependent selection effect.

It is well known that the effective radius of inactive late-type galaxies increases toward shorter wavelengths (e.g., Möllenhoff $\&$ Heidt 2001). This dependence delineates the morphology of SF regions in the galaxies, in the sense that SF (blue bands) occurs in the spiral arms while the old stellar population (red bands) dominates the bulge component. On the other hand, de Grijs (1998) found no change in the effective radius from $B$ - to $K$-band for inactive early-type galaxies (up to Sa spirals). The average effective radii of our BL Lac host galaxy sample are $R_{\mathrm{e}}(U)=6.4 \pm 2.3 \mathrm{kpc}, R_{\mathrm{e}}(B)=7.6 \pm 3.4 \mathrm{kpc}$ and $R_{\mathrm{e}}(V)=7.4 \pm 2.6$ kpc, comparable to those in the $H$-band $\left(R_{\mathrm{e}}(H)=7.8 \pm 4.3 \mathrm{kpc}\right.$; Kotilainen \& Falomo 2004) and in the $R$-band $\left(R_{\mathrm{e}}(R)=9 \pm 5 \mathrm{kpc}\right.$; Falomo \& Kotilainen 1999). In agreement with the result for inactive ellipticals (de Grijs 1998), we find that the effective radius of the early-type AGN host galaxies does not decrease with wavelength. If anything, one can note a slight increasing trend. However, this result should be taken with caution because of the relatively large errors in determining the effective radius depending on the well-known degeneracy between surface brightness and effective radius.

\subsection{Kormendy relation}

According to the Kormendy relation, there is a tight relation between the effective radius $R_{\mathrm{e}}$ and the surface brightness $\mu_{\mathrm{e}}$ (Kormendy 1977; Kormendy \& Djorgovski 1989). This relation is a 2D projection of the 3D Fundamental Plane (e.g., Dressler et al. 1987; Djorgovski \& Davis 1987) that links $R_{\mathrm{e}}$ and $\mu_{\mathrm{e}}$ with the stellar velocity dispersion $\sigma$. This relation, well established for inactive elliptical galaxies and the bulges of spiral galaxies in nearby clusters (e.g., Jörgensen et al. 1993, 1996), is related to the morphology and the dynamical structure of the galaxies and gives important information about their formation processes, indicating underlying regularity within the galaxy populations.

Figure 3 presents the $B$-band Kormendy relation for our sample of $18 \mathrm{BL}$ Lac objects, compared with that for nearby RGs (Govoni et al. 2000), isolated inactive elliptical galaxies (Reda et al. 2005) and inactive ellipticals in the Coma cluster (Jörgensen et al. 1993). The corresponding surface brightnesses $\mu_{\mathrm{e}}$ and effective radii $R_{\mathrm{e}}$ for the BL Lac hosts are given in Table 3. The surface brightnesses of the host galaxies were corrected for Galactic extinction and cosmological dimming $(10 \times \log (1+z))$. Note that the surface brightnesses $\mu_{\mathrm{e}}$ and effective radii $R_{\mathrm{e}}$ refer to the isophote that contains half of the total luminosity of the galaxy. Another commonly used definition for $\mu_{\mathrm{e}}$ 
Table 3. Properties of the host galaxies ${ }^{a}$.

\begin{tabular}{|c|c|c|c|c|c|c|c|c|c|c|c|}
\hline Name & $\begin{array}{c}\text { Filter } \\
(2)\end{array}$ & $\begin{array}{c}A \\
\operatorname{mag} \\
(3)\end{array}$ & (4) & $\begin{array}{l}m_{\text {nuc }} \\
\text { mag } \\
(5)\end{array}$ & $\begin{array}{c}m_{\text {host }} \\
\text { mag } \\
(6)\end{array}$ & $\begin{array}{c}\mu_{\mathrm{e}} \\
\operatorname{mag} \underset{(7)}{\operatorname{arcsec}}{ }^{2}\end{array}$ & $\begin{array}{c}r_{\mathrm{e}} \\
\operatorname{arcsec} \\
(8)\end{array}$ & $\begin{array}{c}R_{\mathrm{e}} \\
\mathrm{kpc} \\
(9)\end{array}$ & $\begin{array}{l}M_{\text {nuc }} \\
\text { mag } \\
(10)\end{array}$ & $\begin{array}{c}M_{\text {host }} \\
\text { mag } \\
(11)\end{array}$ & $N / H$ \\
\hline \multirow{3}{*}{ 1ES 0229+200 } & $U$ & 0.84 & 0.139 & 19.22 & 18.83 & 20.94 & 2.75 & 6.55 & -20.6 & -21.5 & 0.44 \\
\hline & $B$ & 0.71 & & 19.58 & 18.57 & 22.39 & 5.65 & 13.4 & -20.2 & -21.9 & 0.21 \\
\hline & V & 0.54 & & 19.67 & 17.58 & 21.72 & 4.90 & 11.7 & -19.9 & -22.2 & 0.12 \\
\hline PKS 0521-365 & $B$ & 0.25 & 0.055 & 16.58 & 16.17 & 21.50 & 7.60 & 8.02 & -20.6 & -21.3 & 0.52 \\
\hline PKS 0548-322 & $B$ & 0.17 & 0.069 & 17.42 & 16.11 & 22.31 & 9.40 & 12.2 & -20.2 & -21.8 & 0.23 \\
\hline \multirow[t]{3}{*}{$1 \mathrm{H} 0706+591$} & $U$ & 0.50 & 0.125 & 17.67 & 18.55 & 21.90 & 3.60 & 7.83 & -21.6 & -21.1 & 1.6 \\
\hline & $B$ & 0.42 & & 18.38 & 17.86 & 21.21 & 3.50 & 7.63 & -20.8 & -22.0 & 0.33 \\
\hline & $V$ & 0.32 & & 18.10 & 16.57 & 20.40 & 3.65 & 7.96 & -21.0 & -22.8 & 0.19 \\
\hline \multirow[t]{3}{*}{ MRK 421} & $U$ & 0.16 & 0.031 & 13.41 & 15.22 & 21.29 & 7.80 & 4.80 & -22.4 & -20.7 & 4.8 \\
\hline & $B$ & 0.14 & & 14.92 & 15.43 & 21.78 & 8.90 & 5.48 & -20.9 & -20.5 & 1.4 \\
\hline & $H$ & 0.00 & & 15.18 & 14.29 & 16.82 & 6.95 & 4.45 & -20.5 & -24.9 & 0.02 \\
\hline \multirow[t]{2}{*}{ MRK 180} & $U$ & 0.16 & 0.045 & 14.83 & 15.81 & 20.83 & 4.95 & 4.33 & -21.8 & -21.0 & 2.1 \\
\hline & $B$ & 0.14 & & 16.44 & 15.45 & 21.91 & 9.45 & 8.27 & -20.2 & -21.4 & 0.33 \\
\hline 1ES $1212+078$ & $B$ & 0.13 & 0.136 & 19.17 & 18.53 & 22.09 & 3.80 & 8.89 & -19.9 & -21.3 & 0.28 \\
\hline MS $1229.2+6430$ & $B$ & 0.17 & 0.164 & 19.14 & 18.19 & 21.28 & 3.15 & 8.57 & -20.4 & -22.2 & 0.19 \\
\hline 1ES 1255+244 & $B$ & 0.13 & 0.140 & 19.45 & 18.33 & 21.90 & 3.55 & 8.50 & -19.7 & -21.6 & 0.17 \\
\hline \multirow[t]{2}{*}{ PG 1418+546 } & $U$ & 0.09 & 0.152 & 15.95 & & & & & -23.4 & & \\
\hline & $B$ & 0.08 & & 17.20 & 18.14 & 20.25 & 1.90 & 4.87 & -22.1 & -21.9 & 1.2 \\
\hline 1ES $1426+428$ & $B$ & 0.13 & 0.129 & 17.67 & 17.50 & 19.35 & 1.40 & 3.13 & -21.3 & -22.1 & 0.48 \\
\hline 1ES $1440+122$ & $B$ & 0.13 & 0.162 & 18.14 & 19.17 & 22.43 & 3.65 & 9.84 & -21.4 & -21.2 & 1.2 \\
\hline \multirow[t]{2}{*}{ AP LIBRAE } & $U$ & 0.84 & 0.049 & 15.26 & 16.10 & 19.07 & 2.55 & 2.42 & -22.2 & -21.6 & 1.7 \\
\hline & $B$ & 0.71 & & 16.88 & 16.52 & 19.74 & 2.90 & 2.75 & -20.5 & -21.1 & 0.58 \\
\hline MS 1552.1+2020 & $B$ & 0.29 & 0.273 & 19.22 & 19.07 & 20.09 & 3.40 & 13.53 & -21.7 & -23.1 & 0.28 \\
\hline \multirow{3}{*}{ MRK 501} & $U$ & 0.16 & 0.034 & 14.59 & 14.43 & 21.07 & 8.90 & 5.98 & -21.4 & -21.7 & 0.76 \\
\hline & $B$ & 0.13 & & 14.89 & 14.20 & 20.76 & 9.45 & 6.35 & -21.1 & -22.0 & 0.44 \\
\hline & $V$ & 0.10 & & 14.43 & 13.00 & 19.68 & 9.45 & 6.35 & -21.5 & -23.2 & 0.21 \\
\hline \multirow[t]{3}{*}{ I Zw 187} & $U$ & 0.25 & 0.055 & 16.53 & 17.56 & 21.11 & 2.65 & 2.80 & -20.5 & -19.8 & 1.9 \\
\hline & $B$ & 0.21 & & 17.12 & 17.14 & 21.65 & 4.35 & 4.59 & -20.0 & -20.3 & 0.76 \\
\hline & $V$ & 0.16 & & 16.39 & 15.82 & 20.93 & 5.15 & 5.43 & -20.7 & -21.4 & 0.52 \\
\hline \multirow[t]{3}{*}{ 3C 371} & $U$ & 0.45 & 0.051 & 15.33 & 16.17 & 22.13 & 8.95 & 8.80 & -21.9 & -21.2 & 1.9 \\
\hline & $B$ & 0.38 & & 15.86 & 15.98 & 21.12 & 6.15 & 6.05 & -21.3 & -21.4 & 0.91 \\
\hline & $V$ & 0.29 & & 14.98 & 14.94 & 20.12 & 5.60 & 5.51 & -22.1 & -22.2 & 0.91 \\
\hline BL LACERTAE & $B$ & 1.61 & 0.069 & 14.71 & 17.35 & 19.79 & 3.20 & 4.15 & -24.3 & -22.0 & 8.32 \\
\hline
\end{tabular}

${ }^{a}$ Column (1) gives the name of the object; (2) the filter; (3) the interstellar extinction in the $U, B$ - and $V$-bands; (4) the redshift of the object; (5) the apparent nuclear magnitude; (6) the apparent host galaxy magnitude; (7) surface brightness at the effective radius (8) and (9) the apparent and absolute effective radius; (10) the absolute nuclear magnitude; (11) the absolute host galaxy magnitude and (12) the absolute nuclear/host luminosity ratio.

and $r_{\mathrm{e}}$ is to derive these values directly from the de Vaucouleurs fitting of the galaxy, but that definition is model dependent.

The best-fit linear relation for the BL Lac hosts is $\mu_{\mathrm{e}}=$ $3.3 \log R_{\mathrm{e}}(\mathrm{kpc})+18.4 \mathrm{mag} \operatorname{arcsec}^{-2}$. It is somewhat steeper than that for inactive elliptical galaxies and RGs. It is, however, consistent with the relation obtained by Falomo \& Kotilainen (1999) and Kotilainen \& Falomo (2004) for BL Lac hosts in the $R$ and $H$-bands, indicating that the dynamical structure of the hosts does not change with wavelength. Note that one BL Lac object (MS 1552.1+2020, filled triangle in Fig. 3) clearly deviates from the $\mu_{\mathrm{e}}-R_{\mathrm{e}}$-relation of the sample, towards large effective radius and high surface brightness. Note that MS 1552.1+2020 also has one of the bluest host galaxies in the sample $(B-H=3.2$; Table 5), and its displacement from the Kormendy relation may indicate recent SF as suggested for similar cases among inactive ellipticals by Reda et al. (2005). Overall, it seems that BL Lac hosts are not very far from the Fundamental Plane of inactive elliptical galaxies, indicating that they are dynamically similar. It supports the view that BL Lac hosts and normal ellipticals have a similar formation process and that all elliptical galaxies may have experienced a phase of nuclear activity in the past with little influence on the global structure of the galaxy.

\subsection{Host galaxy colours}

The host galaxies of all the BL Lac objects in our sample were previously studied in the optical $R$-band and in the NIR $H$-band (Falomo \& Kotilainen 1999; Kotilainen \& Falomo 2004). The new observations presented here represent the first host galaxy study performed in the blue part of the spectrum. The combination of blue, optical and NIR data allows us to assess the issue of the optical-NIR colours of the BL Lac host galaxies. While the $H$-band is sensitive to old stellar populations, the blue domain of the spectrum is especially important in studying SF and the presence of a young stellar population component in the galaxies.

The integrated rest-frame $U-B, B-V$ and $B-R$ colours of our BL Lac sample are given in Table 5 . The average colours of the BL Lac hosts, compared to those observed in RLQ and RQQ host galaxies (Jahnke et al. 2004), RGs (Govoni et al. 2000), and inactive elliptical galaxies (Peletier et al. 1990; Bower et al. 1992; Colbert et al. 2001), and theoretically predicted for inactive ellipticals ((Fukugita \& Ichikawa 1995); Fioc \& Rocca-Volmerange 1999) are presented in Table 6. Because $U$ band data is available only for eight objects in our sample, we prefer to use the $B-H$ colour as the longest baseline colour. 


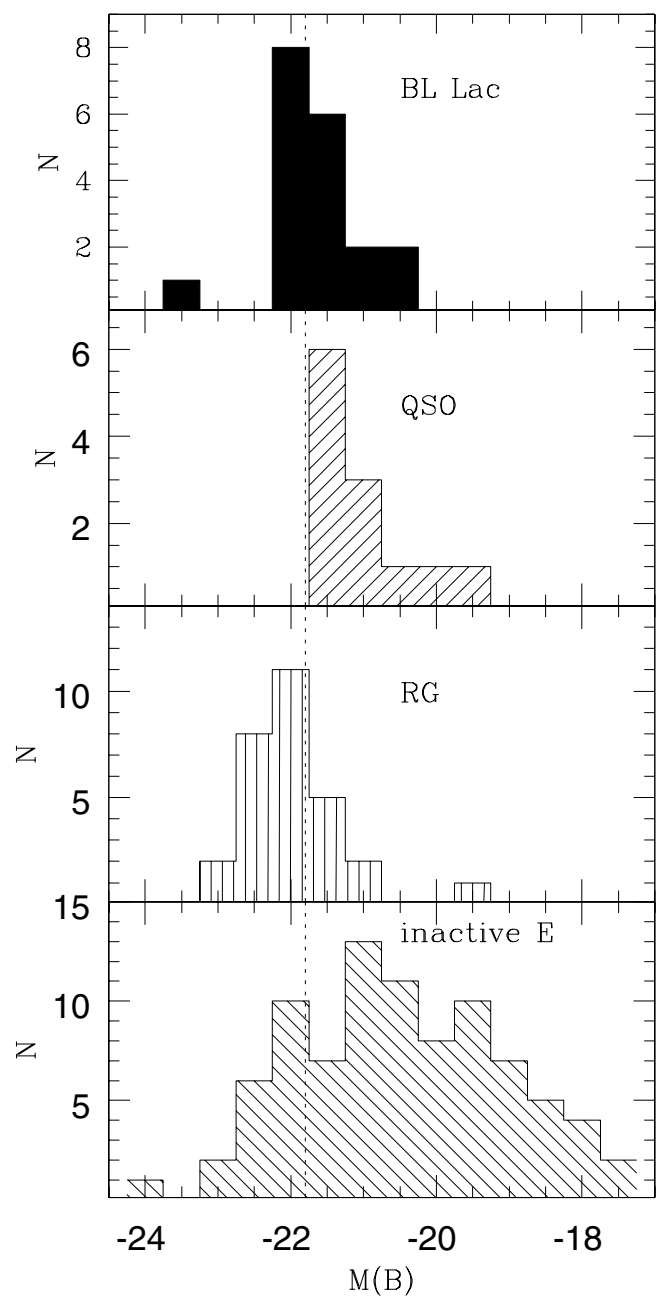

Fig. 2. Histogram of the absolute $B$-band magnitudes of the BL Lac hosts (top panel; this work), low redshift $(z<0.2)$ RLQ and RQQ hosts (second panel; Jahnke et al. 2004), low redshift RGs (third panel; Govoni et al. 2000) and inactive elliptical galaxies (bottom panel; Peletier et al. 1990; Colbert et al. 2001; and Bower et al. 1992). The vertical short dashed line represents the luminosity of the $L^{*}$ galaxies.

The average $B-H$ colour of the BL Lac hosts is $B-H=$ $3.5 \pm 0.5$ that is slightly redder than that of RLQ and RQQ hosts ( $B-H=2.9 \pm 0.3$, Jahnke et al. 2004) but slightly bluer than that of inactive elliptical galaxies $(B-H=3.8 \pm 0.3$, Colbert et al. 2001). The same situation applies when considering the $B-V$ and $B-R$ colours (Table 6). The optical colours of the BL Lac hosts are very similar to those of intermediate/late-type ( $\mathrm{Sb}$ $\mathrm{Sbc}$ ) inactive galaxies with significant ongoing SF (Table 6). Figure 4 shows the distribution of the $B-R$ colour for BL Lac hosts, quasar hosts (Jahnke et al. 2004), RGs (Govoni et al. 2000) and inactive ellipticals (Colbert et al. 2001). BL Lac hosts clearly exhibit a much wider colour distribution and have a significantly bluer host galaxy population that is not present for inactive ellipticals.

It has long been known that the integrated colours of elliptical galaxies become redder toward higher luminosity (mass). (e.g., Bower et al. 1992; Kodama \& Arimoto 1997). This colourmagnitude relation links the properties of the stellar populations of early-type galaxies with their structural properties and provides important information about their formation and evolution. It depends on the combined effects of age and metallicity on the dominant stellar population (more massive galaxies are both older and more metal-rich than less massive galaxies). It is identical in different galaxy clusters, such as in Virgo and Coma clusters (Bower et al. 1992) and overall shows little dependence on environment (Terlevich et al. 2001; Bernardi et al. 2003) and redshift (e.g., Aragon-Salamanca et al. 1993; Kodama et al. 1998; Holden et al. 2004), indicating that massive ellipticals formed in an intense starburst at high redshift followed by passive evolution, with no major SF episodes since $z \sim 2$.

The $B-R$ vs. $R$ and $B-H$ vs. $H$ colour-magnitude diagrams for the BL Lac hosts (this work), low redshift RLQ and RQQ hosts (Jahnke et al. 2004), RGs (Govoni et al. 2000), inactive ellipticals (Peletier et al. 1990; Colbert et al. 2001) and ellipticals in Virgo and Coma clusters (Bower et al. 1992) are presented in Fig. 5. It is evident that the BL Lac hosts do not follow the relatively tight colour-magnitude relation of inactive ellipticals. Instead, they have a significantly broader colour distribution and the majority of them appear to be bluer than inactive ellipticals of similar luminosity. Indeed, such colours are more similar to those found in intermediate/late-type inactive galaxies that have significant recent SF. This result is consistent with the colours of low redshift RGs (Govoni et al. 2000) and quasar hosts (Jahnke et al. 2004). Although this colour difference may indicate that the colour-magnitude relation for elliptical galaxies breaks down at high luminosities as suggested by Govoni et al. (2000) and Kotilainen \& Falomo (2004), note that the elliptical galaxies cover the same luminosity range as the BL Lac hosts. A more likely explanation for the blue colours of BL Lac hosts (and the other AGN hosts in the diagrams) is that they have experienced recent SF. The wide colour distribution indicates a range of timescale since the latest SF episode, such that the bluest hosts have experienced the most recent SF whereas the reddest hosts have experienced little or no SF and are dominated by an old stellar population similar to those in inactive ellipticals. Especially note that there is one BL Lac host with a red $B-R$ colour $(B-R>2.0)$ and two hosts with red $B-H$ colour ( $B-H>4.4)$, redder than any of the inactive ellipticals. From this point of view, the bluest hosts may display evidence from an event that triggered both the nuclear activity and the strong SF. This is consistent with the blue colours $(B-R=1.1 \pm 0.1)$ in RLQ and RQQ hosts (Jahnke et al. 2004). Similarly blue host colours were also found in low redshift quasars by Schade et al. (2000).

\subsection{Colour gradients of the host galaxies}

It is well known that nearby inactive elliptical galaxies do not have uniform colour but instead have negative colour gradients, i.e. they become bluer with increasing radius (e.g., Peletier et al. 1990). These colour gradients have been widely interpreted as due to radial variations in the dust content and/or SF history of the galaxies (e.g., Kim 1989; Peletier et al. 1990; Goudfrooij et al. 1994; Michard 2000). As dust reddening is relatively low in ellipticals, while normal early-type galaxies can be dusty (Tran et al. 2001), most of the colour gradients are usually ascribed to the combined effect of stellar metallicity and age gradients across the galaxies, with the outer regions being younger and/or having lower metallicity.

For the BL Lac host galaxies, we derived the radial $U-B$, $B-R$ and $B-H$ colour gradients using the $R$ - and $H$-band host galaxy luminosity profiles from Kotilainen et al. (1998); Falomo \& Kotilainen (1999); Kotilainen \& Falomo (2004); Scarpa et al. (2000). The $B-R$ colour profiles are presented in Fig. 6 and all colour gradients are presented in Table 5 . Each gradient 
Table 4. Comparison of the average $B$-band host galaxy properties ${ }^{a}$.

\begin{tabular}{|c|c|c|c|c|}
\hline $\begin{array}{l}\text { Name } \\
\text { (1) }\end{array}$ & (2) & $\begin{array}{l}\langle z\rangle \\
\text { (3) }\end{array}$ & $\begin{array}{c}\left\langle M_{B}(\text { host })\right\rangle \\
\text { mag } \\
\text { (4) }\end{array}$ & $\begin{array}{l}\left\langle R_{\mathrm{e}}\right\rangle \\
\mathrm{kpc} \\
(5)\end{array}$ \\
\hline BL Lacs (this work) & 18 & $0.104 \pm 0.064$ & $-21.6 \pm 0.7$ & $7.6 \pm 3.2$ \\
\hline Quasars (Jahnke et al. 2004) & 12 & $0.094 \pm 0.043$ & $-20.9 \pm 0.7$ & $6.0 \pm 2.7$ \\
\hline FR I RGs (Govoni et al. 2000) & 17 & $0.069 \pm 0.031$ & $-22.1 \pm 0.6$ & $15.3 \pm 9.2$ \\
\hline FR II RGs (Govoni et al. 2000) & 6 & $0.050 \pm 0.016$ & $-21.9 \pm 0.6$ & $11.4 \pm 7.7$ \\
\hline
\end{tabular}

${ }^{a}$ Column (1) gives the sample; (2) the number of objects; (3) the average redshift; (4) the average host galaxy magnitude, and (5) the average effective radius $\left(\left\langle R_{\mathrm{e}}\right\rangle\right.$ for $\mathrm{RGs}$ are in $R$-band).

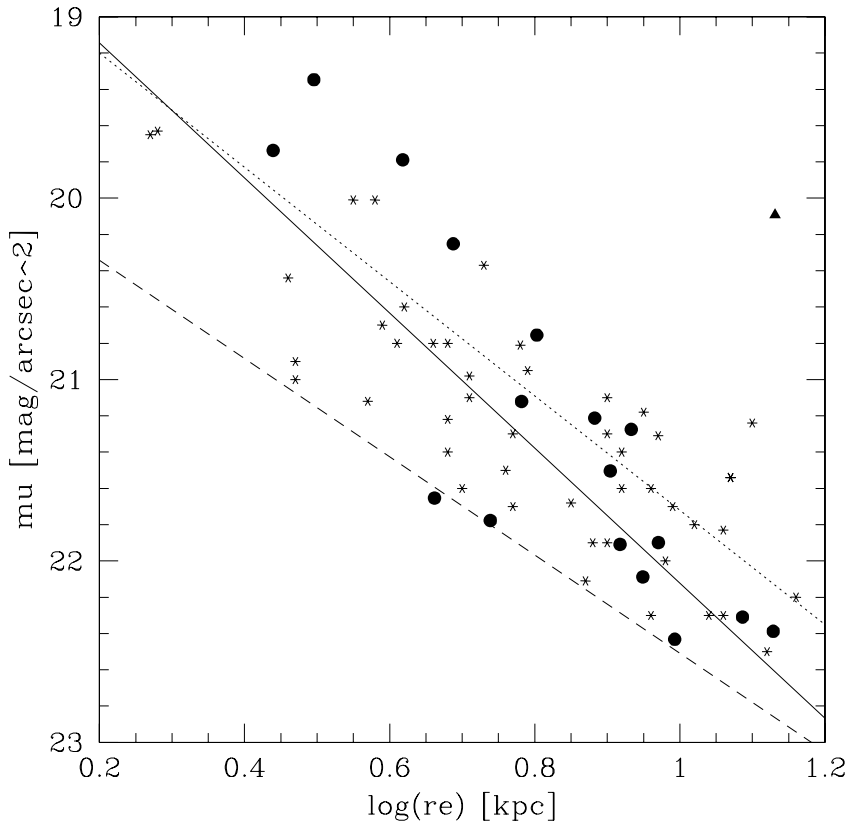

Fig. 3. The $B$-band $\mu_{\mathrm{e}}-R_{\mathrm{e}}$ Kormendy relation for the BL Lac host galaxies (filled symbols), and for inactive elliptical galaxies (asterisks; Jörgensen et al. 1993; Reda et al. 2005). The solid, dotted and long dashed lines represent the best linear fit for BL Lac hosts, inactive ellipticals (Jörgensen et al. 1993; Reda et al. 2005), and low redshift RGs (Fasano et al. 1996), respectively.

was cut at the radius $<2$ arcsec to avoid any contamination of the host galaxy from the bright nucleus. The colour as a function of logarithmic radius is usually well represented by a linear fit. As expected for early-type galaxies, most of the BL Lac host galaxies show a negative colour gradient with average $\Delta(B-R) / \Delta(\log r)=-0.14 \pm 0.75$. The same negative trend was also found for the $R-H$ colour gradients of BL Lacs $(\Delta(R-H) / \Delta(\log r)=-0.38 \pm 0.60$ by Kotilainen \& Falomo 2004). The amplitude of the $B-R$ colour gradients of the BL Lac hosts is consistent with the average $B-R$ gradient of RGs $\Delta(B-R) / \Delta(\log r)=-0.16 \pm 0.17$ (Govoni et al. 2000) and inactive ellipticals $\Delta(B-R) / \Delta(\log r)=-0.09 \pm 0.07$ (Peletier et al. 1990), but the distribution is much wider than that of the RGs and inactive ellipticals. A similar distribution was also obtained for the $R-H$ gradient of BL Lac hosts by Kotilainen \& Falomo (2004).

However, some BL Lac host galaxies show little evidence of colour variation or even have an inverted (positive) colour gradient, the steepest of them in PKS 0548-322, 1ES 1426+428 and MRK 501. A similarly steep positive $R-H$ colour gradient for
1ES1426+428 was observed by Kotilainen \& Falomo (2004). Since the $B-H$ colour is more sensitive to the dust content than the $R-H$ colour, this indicates that the positive $B-R$ and $R-H$ gradients of this target are due to radial variations in the dust content of its host galaxy. Indeed, recent HST observations of RGs (e.g., Martel et al. 2000; Tremblay et al. 2007) have shown that there is a significant number of RGs that contain dust in a variety of spatial distributions, such as circumnuclear disks and dust lanes at kpc scales. On the other hand, dust is not a reasonable explanation for e.g. MRK 501 which has a positive $B-R$ but a negative $R-H$ gradient. In this case, the inverted profiles indicates SF in the inner region of the host galaxy because the host galaxy is well resolved. That indication is also supported by recent spectroscopic observations of ongoing SF in the nuclear regions of a BL Lac object (PKS 2005-489; Bressan et al. 2006).

Figure 7 shows the $U-B$ colour profiles for the eight BL Lac host galaxies for which we have available data (Table 5). All the host galaxies, except one (3C 371), have a positive colour gradient. 3C 371 has a signature of recent interaction (Nilsson et al. 1997) which might be the reason for the negative $U-B$ colour gradient. The average colour gradient for all eight objects is $\Delta(U-B) / \Delta(\log r)=0.28 \pm 0.46$, although for four objects the gradient is very flat.

\subsection{Stellar population model fits}

The $U B V$ colours combined with the previously observed $R$ - and $H$-band data can be used in conjunction with stellar synthesis population models to estimate the approximate ages of the recent SF episodes in the BL Lac host galaxies. In this context, the $U$ and $B$-bands are especially important as they provide photometry shortward of the $4000 \AA$ break, without which only approximate average ages can be determined. Note also that dust reddening has little effect, since any reddening only would imply even bluer intrinsic colours. The SED modelling utilizes all the colour information simultaneously by fitting the SED of evolution synthesis model to calibrated fluxes of the host galaxies at different wavelengths. Each wavelength band represents one data point in the SED of the object. Comparing fits made for different model spectra gives an estimate of the ages of the dominant stellar population components in the host galaxy. Such SED modelling has been performed by Jahnke et al. (2004) for low redshift RLQ and RQQ hosts. For the analysis we used the PEGASE2 evolutionary model (Fioc \& Rocca-Volmerange 1997) and a single stellar population (SSP) model of single metallicity because the age-metallicity degeneracy in models cannot be resolved with multicolour data only. We used instantanous burst models which assume that the young stellar population is formed in a short burst with an IMF and evolves passively thereafter. 
Table 5. Colours and colour gradients of the BL Lac host galaxies ${ }^{a}$.

\begin{tabular}{lccccccc}
\hline \hline Name & $U-B$ & $B-V$ & $B-R$ & $R-H$ & $\Delta(U-B) / \Delta(\log r)$ & $\Delta(B-R) / \Delta(\log r)$ & $\Delta(B-H) / \Delta(\log r)$ \\
& & & & & & \\
$(1)$ & $(2)$ & $(3)$ & $(4)$ & $(5)$ & $(6)$ & $(7)$ & -0.17 \\
\hline 1ES 0229+200 & 0.4 & 0.3 & 2.0 & 2.5 & 0.92 & -0.44 & -1.66 \\
PKS 0521-365 & & 0.9 & 0.9 & 2.3 & & -1.02 & -0.90 \\
PKS 0548-322 & & & 1.1 & 2.1 & & 1.10 & -0.12 \\
1H 0706+591 & 0.9 & 0.8 & 1.3 & 2.2 & -0.01 & 0.17 & -0.31 \\
MRK 421 & -0.2 & & 2.2 & 2.2 & 0.17 & -0.91 & -0.85 \\
MRK 180 & 0.4 & & 0.5 & 2.7 & 0.75 & -1.24 & -0.74 \\
1ES 1212+078 & & & 1.9 & 2.2 & & 0.48 & -0.91 \\
MS 1229.2+6430 & & & 1.3 & 0.9 & & -0.12 & 0.86 \\
1ES 1255+244 & & & 0.9 & 2.1 & & -1.08 & -0.11 \\
PG 1418+546 & & & 1.4 & 2.2 & & -0.21 & -0.31 \\
1ES 1426+428 & & & 0.8 & 2.5 & & 0.90 & -0.37 \\
1ES 1440+122 & & & 1.6 & 2.4 & & 0.16 & -0.62 \\
AP LIBRAE & -0.5 & & 1.1 & 2.2 & 0.17 & 0.43 & -0.54 \\
MS 1552.1+2020 & & & 0.5 & 2.7 & & -0.41 & -1.51 \\
MRK 501 & 0.4 & 1.2 & 1.1 & 2.4 & 0.01 & 0.64 & -1.70 \\
I Zw 187 & 0.5 & 1.1 & 1.2 & 2.3 & 0.63 & -0.32 & -1.26 \\
3C 371 & 0.2 & 0.8 & 1.5 & 1.9 & -0.44 & 0.63 & \\
BL LACERTAE & & & 1.0 & 2.4 & & & \\
\hline
\end{tabular}

${ }^{a}$ Column (1) gives the name of the object; (2) the $U-B$ colour; (3) the $B-V$ colour; (4) the $B-R$ colour; (5) the $R-H$ colour (from Kotilainen $\&$ Falomo 2004); (6) the $U-B$ colour gradient; (7) the $B-R$ colour gradient and (8) the $B-H$ colour gradient of the host galaxy.

Table 6. Average colours of the host galaxies ${ }^{a}$.

\begin{tabular}{|c|c|c|c|c|c|}
\hline The sample & $U-B$ & $B-V$ & $B-R$ & $R-H$ & $B-H$ \\
\hline (1) & (2) & (3) & (4) & (5) & (6) \\
\hline BL Lacs (this work) & $0.3 \pm 0.4$ & $0.8 \pm 0.2$ & $1.2 \pm 0.5$ & $2.2 \pm 0.4$ & $3.5 \pm 0.5$ \\
\hline Quasars (Jahnke et al. 2004) & & $0.5 \pm 0.1$ & $1.1 \pm 0.1$ & $1.9 \pm 0.2$ & $2.9 \pm 0.3$ \\
\hline RGs (Govoni et al. 2000) & & & $1.4 \pm 0.2$ & & \\
\hline Ellipticals (Peletier et al. 1990) & $0.6 \pm 0.1$ & & $1.6 \pm 0.1$ & & \\
\hline Ellipticals (Bower et al. 1992) & & & & & $3.8 \pm 0.2$ \\
\hline Ellipticals (Colbert et al. 2001) & & & $1.5 \pm 0.2$ & & \\
\hline Ellipticals (Fukugita \& Ichikawa 1995) & & 1.0 & 1.6 & & \\
\hline Ellipticals (Fioc \& Rocca-Volmerange 1999) & & & & 2.2 & 3.8 \\
\hline Spirals (Sb) (Fukugita \& Ichikawa 1995; Fioc \& Rocca-Volmerange 1999) & & 0.7 & 1.2 & 2.1 & 3.3 \\
\hline
\end{tabular}

${ }^{a}$ Column (1) gives the sample; and Cols. (2)-(6) the average $U-B, B-V, B-R, R-H$ and $B-H$ colour, respectively.

Synthesized galaxy spectra composed from libraries of stellar spectra can be used to derive the colours of the galaxies over a large wavelength range from UV to NIR for different ages and metallicities. For generating the synthetic spectra we used the Scalo (1990) initial mass function, solar metallicity and five different model ages $(0.1,0.7,2,6$ and $14 \mathrm{Gyr})$, to allow a direct comparison with the results of Jahnke et al. (2004).

From the synthesized spectra we have derived the $U-B, B-V$, $B-R, R-H$ and $B-H$ colours for each model age and compared those with the observed colours of each BL Lac using an iterative least squares fit, with age as the only free parameter. The results of the fit are presented in Table 7, where the best fitting model is marked as 1 and the poorest fitting model as 3 .

The fit results indicate that the best fit models are generally consistent with a young/intermediate age stellar population. Only for two BL Lacs (1ES 0229+200 and MRK 421) is the best fitting model older than 2 Gyr and is most consistent with the 6 Gyr model. Note that these objects also have the reddest host galaxies in the sample $(B-H \sim 4.4$, see Table 5$)$. The model fit is thus consistent with the host galaxy colours, suggesting that the host galaxies of these objects are dominated by an old stellar population, with no evidence for a young population. Eight objects are best fit with the 2 Gyr model, while the remaining eight objects in the sample require an even younger model, $0.7 \mathrm{Gyr}$, and in the case of MS 1552.1+2020, the $0.1 \mathrm{Gyr}$ model. Note that MS 1552.1+2020 deviates from the sample also in the Kormendy relation (see above).

These results are in excellent agreement with similarly young stellar populations found by Jahnke et al. (2004) for RLQ and RQQ host galaxies obtained from population synthesis models. They found the 2 Gyr model to best fit their data in 12 out of their 19 objects, and an even younger population $(0.7 \mathrm{Gyr})$ in five objects, while an older population (6 Gyr) was preferred in only two objects. Note that, as is the case for the quasar hosts (Jahnke et al. 2004), none of the elliptical BL Lac hosts are best modeled with the 14 Gyr model, i.e. a very old, evolved population as would be expected for early-type host galaxies. On the other hand, for both types of AGN hosts, there is also little evidence for massive ongoing starbursts with a significant very young population (age $\ll 1 \mathrm{Gyr}$ ). Together these results are in good agreement with the colour information (Sect. 3.3) and strongly support the idea that the blue colours of the early-type 

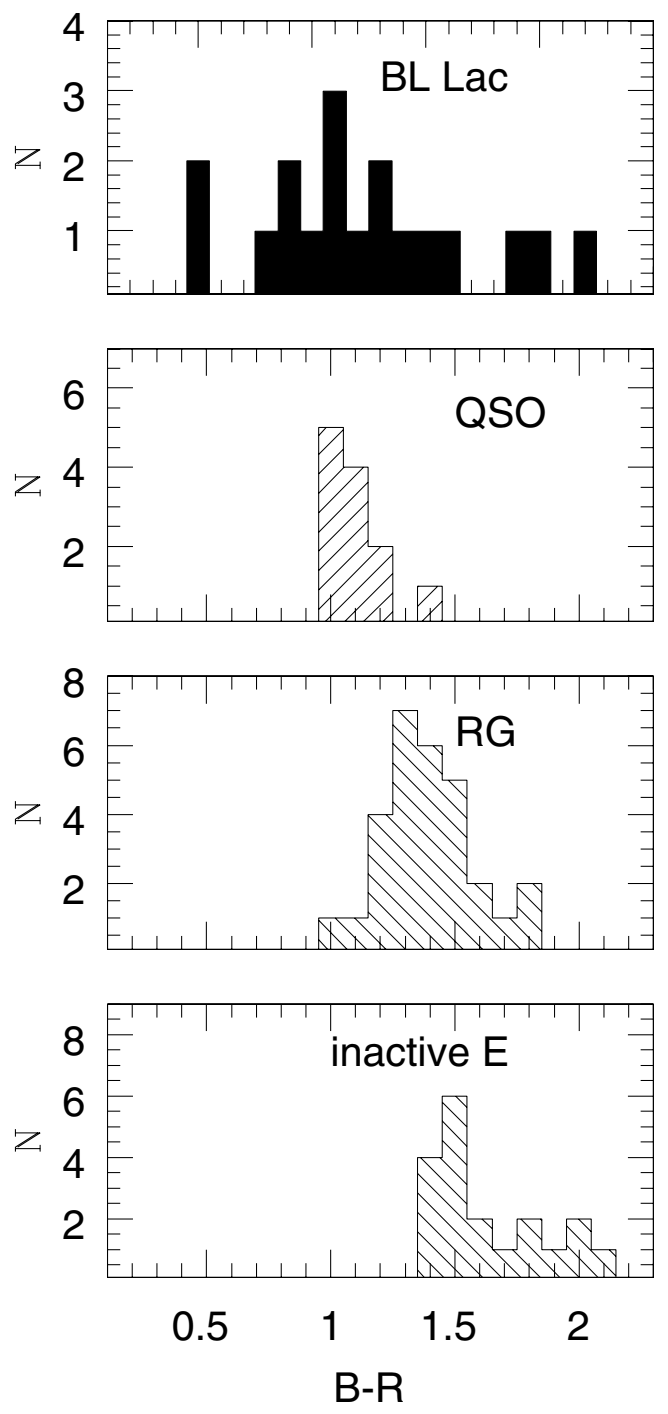

Fig. 4. The distribution of the $B-R$ colour for BL Lac hosts (top panel; this work), low redshift $(z<0.2)$ RLQ and RQQ hosts (second panel; Jahnke et al. 2004), low redshift RGs (third panel; Govoni et al. 2000) and inactive elliptical galaxies (bottom panel; Colbert et al. 2001; Peletier et al. 1990).

host galaxies of moderately luminous AGN are caused by them having experienced a relatively recent SF episode. Further support for this conclusion is provided by spectroscopic evidence for young/intermediate age populations in AGN hosts (e.g., Nolan et al. 2001; Raimann et al. 2005). On the other hand, both the predominantly late-type hosts of low luminosity AGN (e.g., Kotilainen \& Ward 1994; Schade et al. 2000; Jahnke et al. 2004) and the elliptical hosts of very luminous AGN (e.g., Dunlop et al. 2003) exhibit similar colours to their inactive counterparts.

The blue colours and steep colour gradients of early-type AGN hosts found in an increasing number of studies are most likely caused by a young stellar population, and indicate a link between SF and the onset of the nuclear activity, both likely triggered by a tidal interaction or a minor or major merging event. However, the lack of obvious signs of interaction (companion galaxies, tidal tails, disturbed morphology) in the close environment of the large majority of the host galaxies may require a significant time delay (at least hundreds of Myr) between the event with associated SF episodes and the start of the nuclear activity. Such a time delay is indeed predicted by simulations of
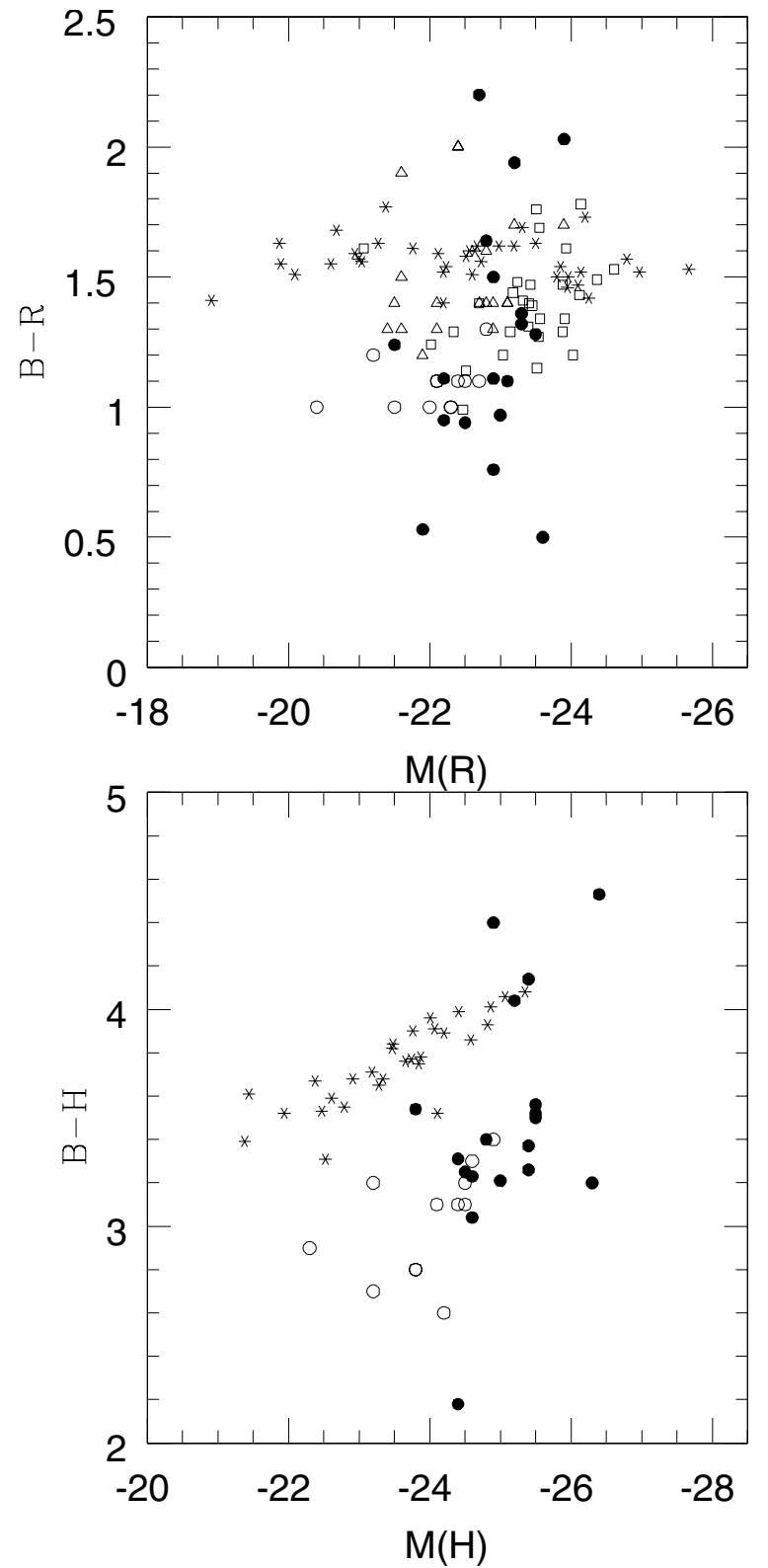

Fig. 5. Upper: the $B-R$ vs. $R$ colour-magnitude diagram for BL Lac host galaxies (filled circles), RLQ and RQQ hosts (open circles; Jahnke et al. 2004), RGs (open squares; Govoni et al. 2000) and inactive early-type galaxies (asterisks; Peletier et al. 1990; Colbert et al. 2001). Lower: the $B-H$ vs. $H$ colour-magnitude diagram for BL Lac host galaxies and comparison samples. Open circles are from Jahnke et al. (2004) and asterisks from Bower et al. (1992).

gas reaching the galaxy center after a merger/interaction (e.g., Lin et al. 1988).

\section{Conclusions}

We have presented $B$-band imaging of a sample of 18 low redshift $(z<0.3)$ BL Lac objects for which their host galaxies were previously resolved in the optical $R$ - and NIR $H$-bands. Subsamples consisting of nine and five BL Lacs were also imaged in the $U$ - and $V$-bands, respectively. All the BL Lac objects were clearly resolved in the $B$ - and $V$-bands while $8 / 9$ objects were resolved in the $U$-band. All the host galaxies are well described by an elliptical de Vaucouleurs model. These data 

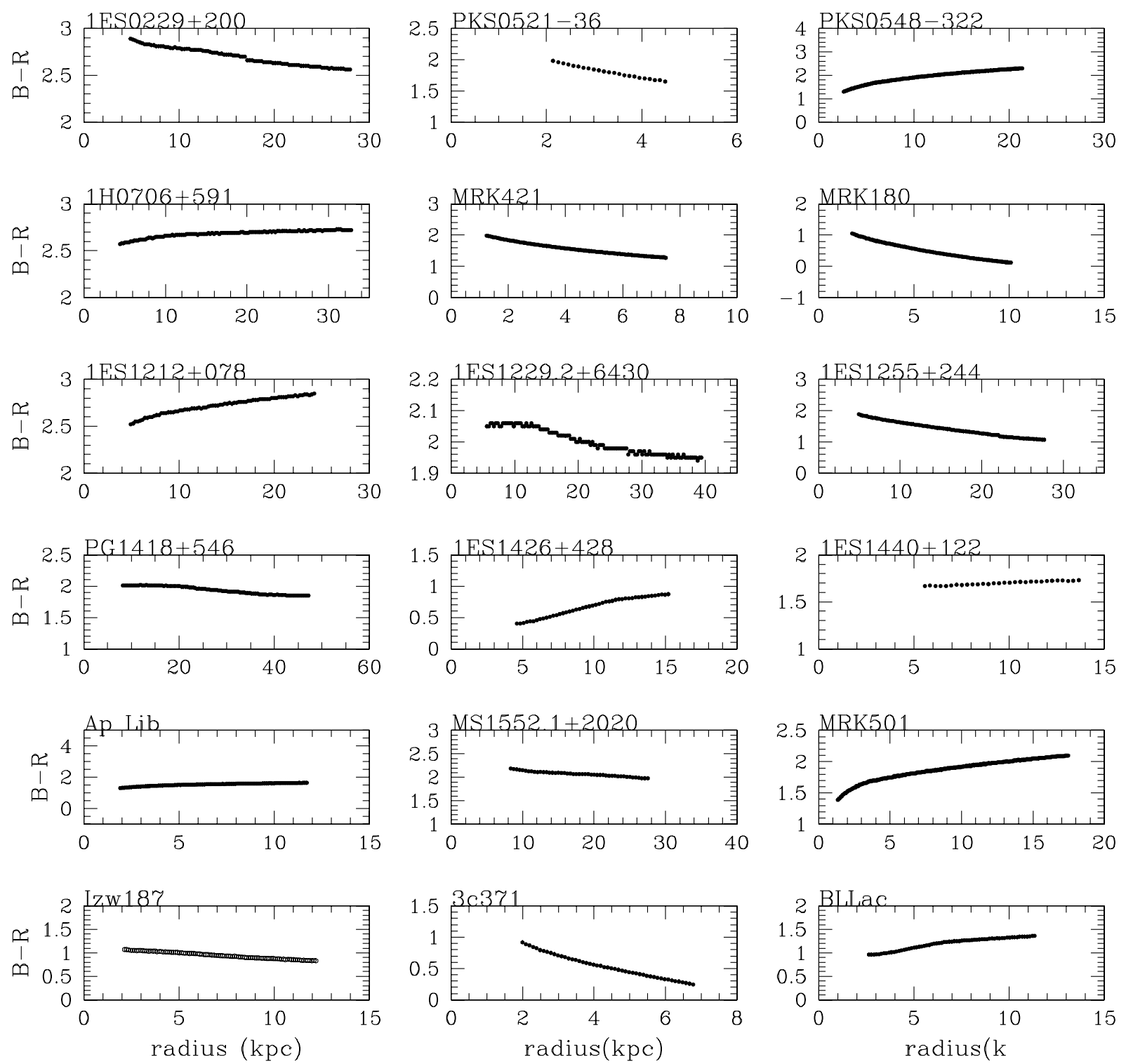

Fig. 6. The $B$ - $R$ colour profiles for the BL Lac host galaxies, derived using the $R$-band data from Falomo \& Kotilainen (1999); Scarpa et al. (2000) and Kotilainen \& Falomo (2004).
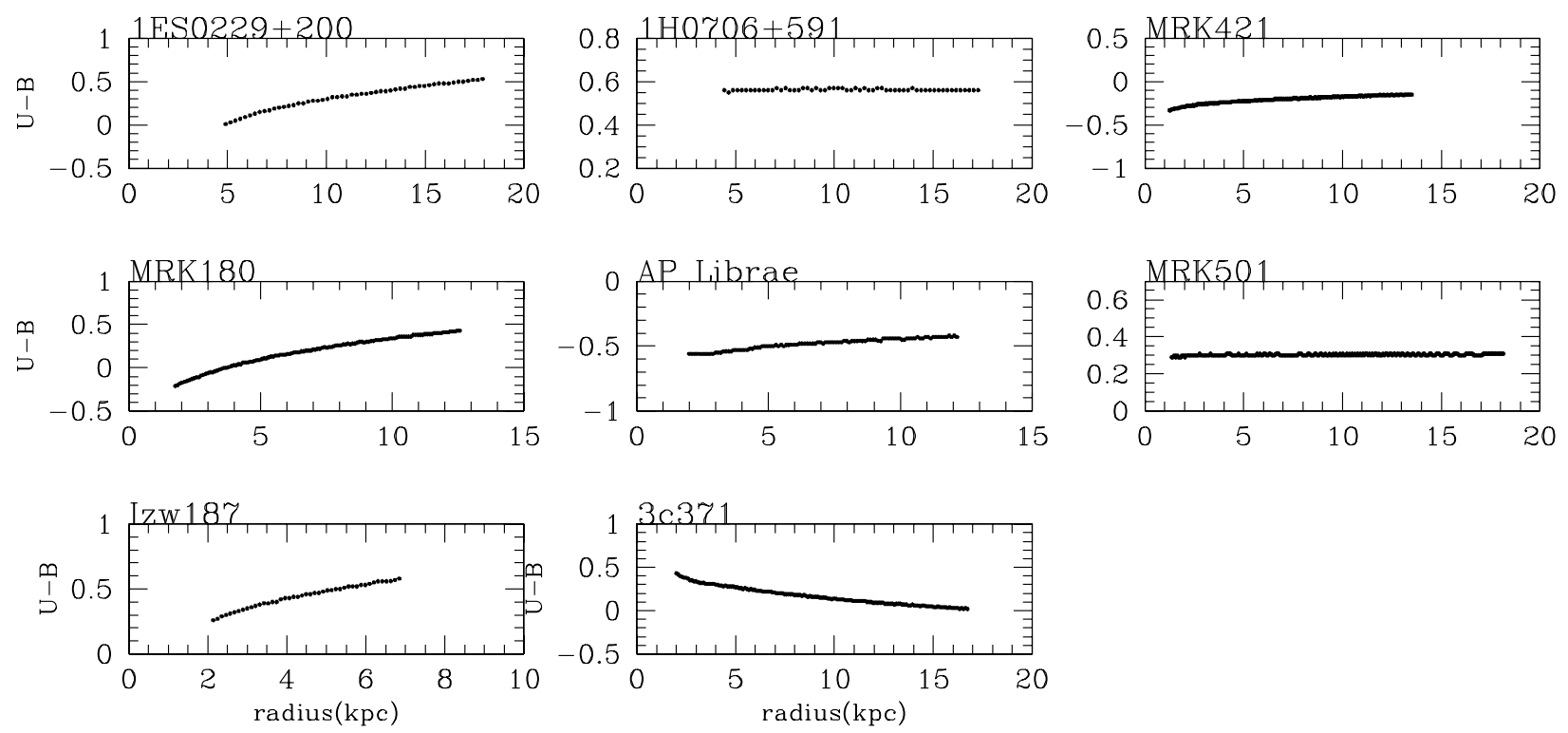

Fig. 7. The $U-B$ colour profiles for BL Lac hosts. 

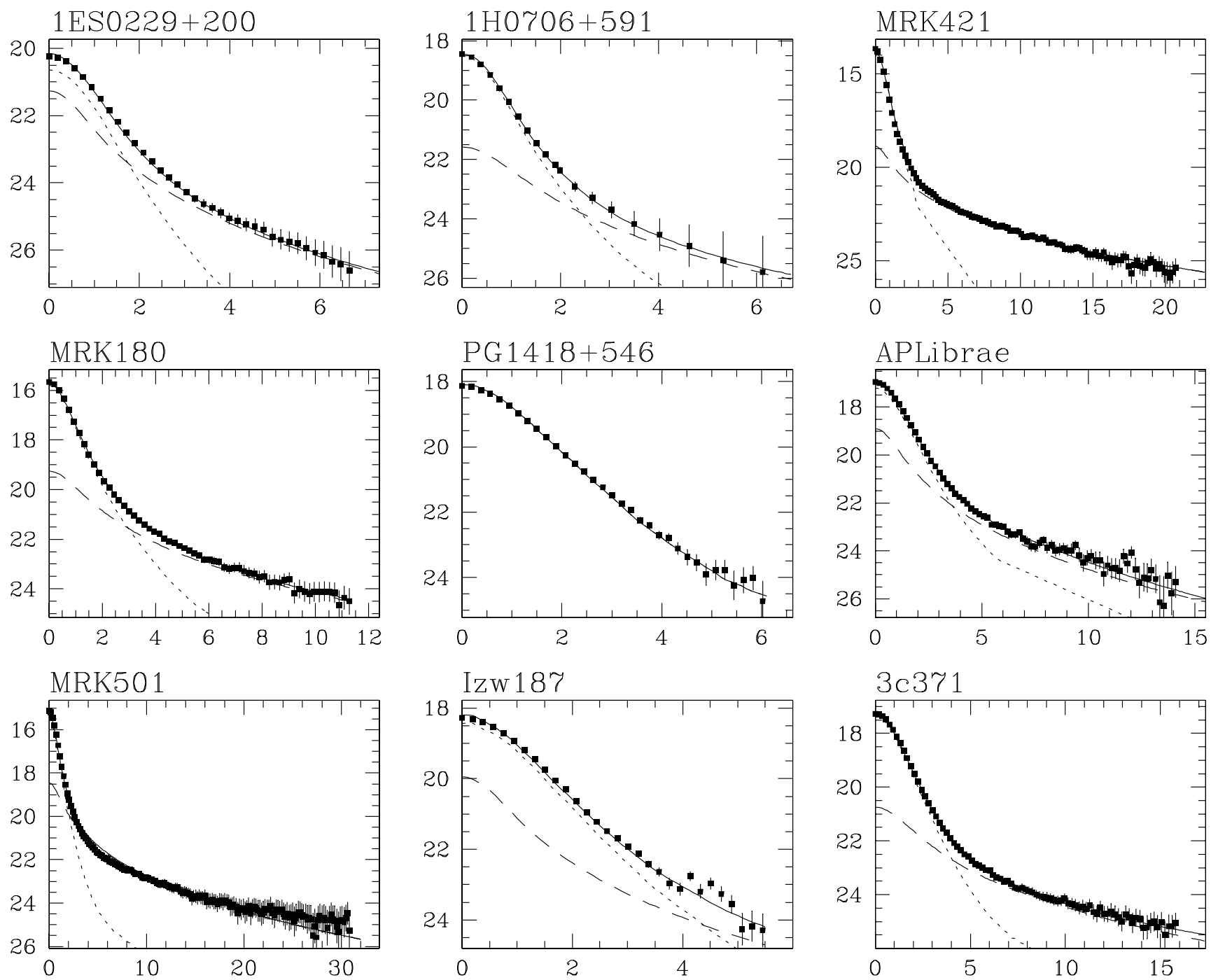

Fig. 8. The observed $U$-band radial luminosity profiles (solid points with error bars) for each BL Lac, overlaid with the PSF model (dotted line), the de Vaucouleurs $r^{1 / 4}$ model (long-dashed line) and the fitted PSF+host galaxy model profile (solid line). The $X$-axis is in arcsec and the $Y$-axis in $\operatorname{mag} \operatorname{arcsec}^{-2}$.

Table 7. Colours of the single SSP fit compared to the colours of the BL Lac sample. 1 represents the best and 3 the worst fit.

\begin{tabular}{llllll}
\hline \hline Name & 0.1 & 0.7 & 2.0 & 6.0 & 14.0 \\
& Gyr & Gyr & Gyr & Gyr & Gyr \\
\hline 1ES 0229+200 & & & 3 & 1 & 2 \\
PKS 0521-365 & 3 & 1 & 2 & & \\
PKS 0548-322 & 2 & 1 & 3 & & \\
1H 0706+591 & & 2 & 1 & 3 & \\
MRK 421 & & & 2 & 1 & 3 \\
MRK 180 & 3 & 1 & 2 & & \\
1ES 1212+078 & & & 1 & 2 & 3 \\
MS 1229.2+6430 & 2 & 1 & 3 & & \\
1ES 1255+244 & 2 & 1 & 3 & & \\
PG 1418+546 & & 2 & 1 & 3 & \\
1ES 1426+428 & 3 & 2 & 1 & & \\
1ES 1440+122 & & & 1 & 2 & 3 \\
AP LIBRAE & 3 & 1 & 2 & & \\
MS 1552.1+2020 & 1 & 2 & 3 & & \\
MRK 501 & & 2 & 1 & 3 & \\
I Zw 187 & & 2 & 1 & 3 & \\
3c371 & & 2 & 1 & 3 & \\
BL LACERTAE & 3 & 1 & 2 & & \\
\hline
\end{tabular}

are combined with previous optical and NIR data to study the optical-NIR colours and colour gradients of the host galaxies.

BL Lac host galaxies are luminous (massive) and large elliptical galaxies having an average $B$-band absolute magnitude $M_{B}=-21.6 \pm 0.7$ and average effective radius $R_{\mathrm{e}}=7.6 \pm 3.2 \mathrm{kpc}$. They are indistinguishable from low redshift radio galaxies but are brighter than inactive elliptical galaxies. The $B$-band Kormendy relation for BL Lac hosts is slightly steeper than that of RGs but does not deviate significantly from the Fundamental Plane of inactive elliptical galaxies. This indicates that BL Lac hosts are dynamically similar to normal ellipticals and that the active nuclear phase does not have any significant influence on the dynamical structure of the galaxy. Thus it is possible that all massive galaxies can experience an AGN phase.

The distributions of the integrated blue/NIR colours $(B-$ $H=3.5 \pm 0.5 ; B-R=1.2 \pm 0.5)$ and colour gradients $(\Delta(B-R) / \Delta(\log r)=-0.14 \pm 0.75)$ of the BL Lac hosts are much wider than those for normal ellipticals with old stellar populations, and many BL Lac objects have bluer hosts and/or steeper colour gradients than those in normal ellipticals. The blue colours and steep colour gradients are most likely caused by a young stellar population, and indicate a link between SF 
caused by an interaction/merging event and the onset of the nuclear activity. Many targets have inverted (positive) colour gradients which in some cases, e.g. 1ES 1426+428, are most likely caused by radial variations in the dust content of the host galaxy, but in some other cases may suggest star formation in the inner regions of the host galaxy. This result is corroborated by stellar population modelling, indicating young/intermediate age populations in the majority of the sample, in agreement with low redshift quasar hosts. However, the lack of obvious signs of interaction may require a significant time delay between the event with associated SF episodes and the start of the nuclear activity.

Future work in this area should address the correlation between colour and statistics of companion galaxies and morphological disturbances as an indicator for the interaction, for well defined samples of AGN hosts and inactive galaxies. In a forthcoming paper, we shall present NIR spectroscopy of BL Lac hosts and RGs with blue colours, to analyze their stellar content and SF properties, based on emission and absorption line diagnostics, in more detail than is available from imaging.

Acknowledgements. Based on observations made with the Nordic Optica Telescope, operated on the island of La Palma jointly by Denmark, Finland, Iceland, Norway, and Sweden, in the Spanish Observatorio del Roque de los Muchachos of the Instituto de Astrofisica de Canarias. This work was supported by the Italian Ministry for University and Research (MIUR) under COFIN 2002/27145, ASI-IR 115 and ASI-IR 35, ASI-IR 73 and by the Academy of Finland (projects 8201017 and 8107775). This research has made use of the NASA/IPAC Extragalactic Database (NED) which is operated by the Jet Propulsion Laboratory, California Institute of Technology, under contract with the National Aeronautics and Space Administration. We thank Valentin Ivanov for obtaining for us the observations with NTT/EMMI.

\section{References}

Antonucci, R., \& Ulvestad, J. 1985, ApJ, 294, 158

Aragon-Salamanca, A., Ellis, R., Couch, W., \& Carter, D. 1993, MNRAS, 262, 764

Bernardi, M., Sheth, R., Annis, J., et al. 2003, AJ, 125, 1882

Blandford, R., \& Rees, M. 1978, in Pittsburgh Conference on BL Lac Objects, ed. A. N. Wolfe (University of Pittsburgh Press), 328

Bower, R., Lucey, J., \& Ellis, R. 1992, MNRAS, 254, 589

Bressan, A., Falomo, R., Valdes, J., \& Rampazzo, R. 2006, ApJ, 645, L101

Cassaro, P., Stanghellini, C., Bondi, M., et al. 1999, A\&AS, 139, 601

Cheung, C., Urry, C., Scarpa, R., \& Giavalisco, M. 2003, ApJ, 599, 155

Colbert, J., Mulchaey, J., \& Zabludoff, A. 2001, AJ, 121, 808

de Grijs, R. 1998, MNRAS, 299, 595

Djorgovski, S., \& Davis, M. 1987, ApJ, 313, 59

Dressler, A., Lynden-Bell, D., Burstein, D., et al. 1987, ApJ, 313, 42
Dunlop, J., McLure, R., Kukula, M., et al. 2003, MNRAS, 340, 1095 Falomo, R., \& Kotilainen, J. 1999, A\&A, 352, 85

Fasano, G., Falomo, R., \& Scarpa, R. 1996, A\&A, 282, 40

Fioc, M., \& Rocca-Volmerange, B. 1997, A\&A, 326, 950

Fioc, M., \& Rocca-Volmerange, B. 1999, AA, 351, 869

Fukugita, M., Shimasaku, K., \& Ichikawa, T. 1995, PASP, 107, 945

Gardner, J., Sharples, R., Frenk, C., \& Carrasco, B. 1997, ApJ, 480, L99

Goudfrooij, P., Hansen, L., \& Jorgensen, H. A. 1994, A\&AS, 104, 179

Govoni, F., Falomo, R., Fasano, G., \& Scarpa, R. 2000, A\&A, 353, 507

Heidt, J., Tröller, M., Nilsson, K., et al. 2004, A\&A, 418, 813

Holden, B., Dtanford, S., Eisenhardt, P., \& Dickinson, M. 2004, AJ, 127, 2484

Jahnke, K., Kuhlbrodt, B., \& Wisotzki, L. 2004, MNRAS, 352, 399

Jörgensen, I., Franx, M., \& Kjaergaard, P. 1993, ApJ, 411, 34

Jörgensen, I., Franx, M., \& Kjaergaard, P. 1996, MNRAS, 280, 167

Kim, D. 1989, ApJ, 346, 653

Kodama, T., \& Arimoto, N. 1997, A\&A, 320, 41

Kodama, T., Arimoto, N., Barger, A., \& Aragón-Salamanca, A. 1998, A\&A, 334, 99

Kollgaard, R., Wardle, J., Roberts, D., \& Gabuzda, D. 1992, AJ, 104, 1687

Kormendy, J. 1977, ApJ, 218, 333

Kormendy, J., \& Djorgovski, S. 1989, ARA\&A, 27, 235

Kotilainen, J., \& Falomo, R. 2004, A\&A, 424, 107

Kotilainen, J., \& Ward, M. 1994, MNRAS, 266, 953

Kotilainen, J., Falomo, R., \& Scarpa, R. 1998, A\&A, 336, 479

Kotilainen, J., Hyvönen, T., \& Falomo, R. 2005, A\&A, 440, 831

Landolt, A. 1992, AJ, 104, 340

Lin, D., Pringle, J., \& Rees, M. 1988, ApJ, 328, 103

Martel, A., Turner, N., Sparks, W., \& Baum, S. 2000, ApJS, 130, 267

Michard, R. 2000, A\&A, 360, 85

Möllenhoff, C., \& Heidt, J. 2001, A\&A, 368, 16

Nakamura, O., Fukugita, M., Yasuda, N., et al. 2003, AJ, 125, 1682

Nilsson, K., Heidt, J., Pursimo, T., et al. 1997, ApJ, 484, L107

Nilsson, K., Pursimo, T., Heidt, J., et al. 2003, A\&A, 400, 95

Nolan, L., Dunlop, J., Kukula, M., Hughes, D., \& Boroson, T. 2001, MNRAS, 323, 308

Örndahl, E., Rönnback, J., \& van Groningen, E. 2003, A\&A, 404, 883

Peletier, R., Valentijn, E., \& Jameson, R. 1990, A\&A, 233, 62

Poggianti, B. 1997, A\&AS, 122, 399

Raimann, D., Storchi-Bergman, T., Quintana, H., Hunstead, R., \& Wisotzki, L. 2005, MNRAS, 364, 1239

Reda, F., Forbes, D., \& Hau, G. 2005, MNRAS, 360, 693

Sanchez, S., Jahnke, K., Wisotzki, L., et al. 2004, ApJ, 614, 586

Scalo, J. 1990, Fund. Cosm. Phys, 11, 1

Scarpa, R., Urry, C., Padovani, P., Calzetti, D., \& O'Dowd, M. 2000, ApJ, 544, 258

Schade, D., Boyle, B., \& Letawsky, M. 2000, MNRAS, 315, 498

Stanghellini, C., Dallacasa, D., Bondi, M., \& della Ceca, R. 1997, A\&A, 325, 911

Terlevich, A., Caldwell, N., \& Bower, R. 2001, MNRAS, 326, 1547

Tran, H., Tsvetanov, Z., Ford, H., et al. 2001, AJ, 121, 2928

Tremblay, G., Chiaberge, M., Donzelli, C., et al. 2007, ApJ, 666, 109

Urry, C., \& Padovani, P. 1995, PASP, 107, 803

Urry, C., Scarpa, R., O’Dowd, M., et al. 2000, ApJ, 532, 816 
T. Hyvönen et al.: The stellar content of low redshift BL Lac host galaxies, Online Material p 1

\section{Online Material}


T. Hyvönen et al.: The stellar content of low redshift BL Lac host galaxies, Online Material p 2
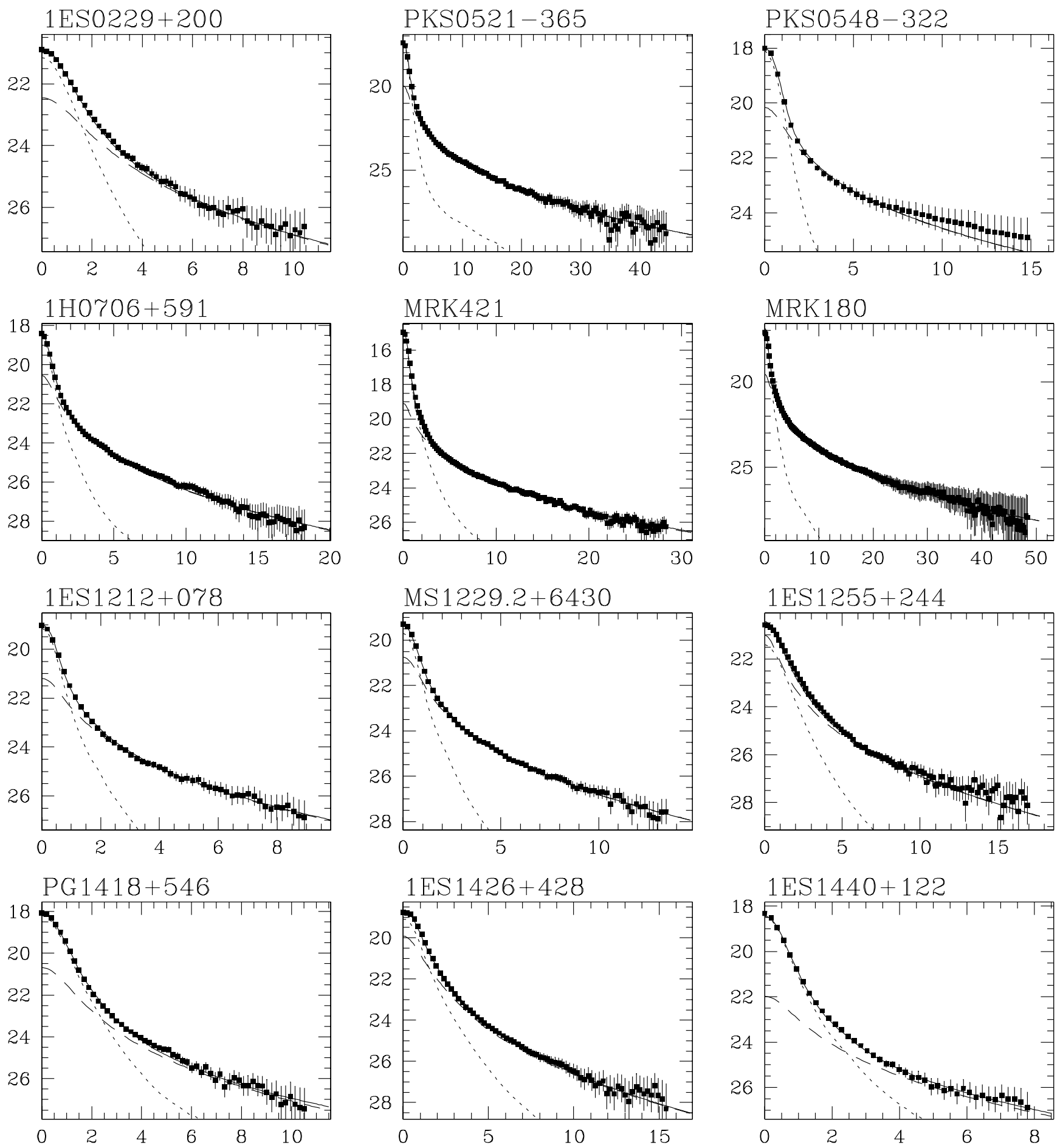

Fig. 9. The observed $B$-band radial luminosity profiles and model profiles. For explanation, see the caption of Fig. 8. 
T. Hyvönen et al.: The stellar content of low redshift BL Lac host galaxies, Online Material p 3
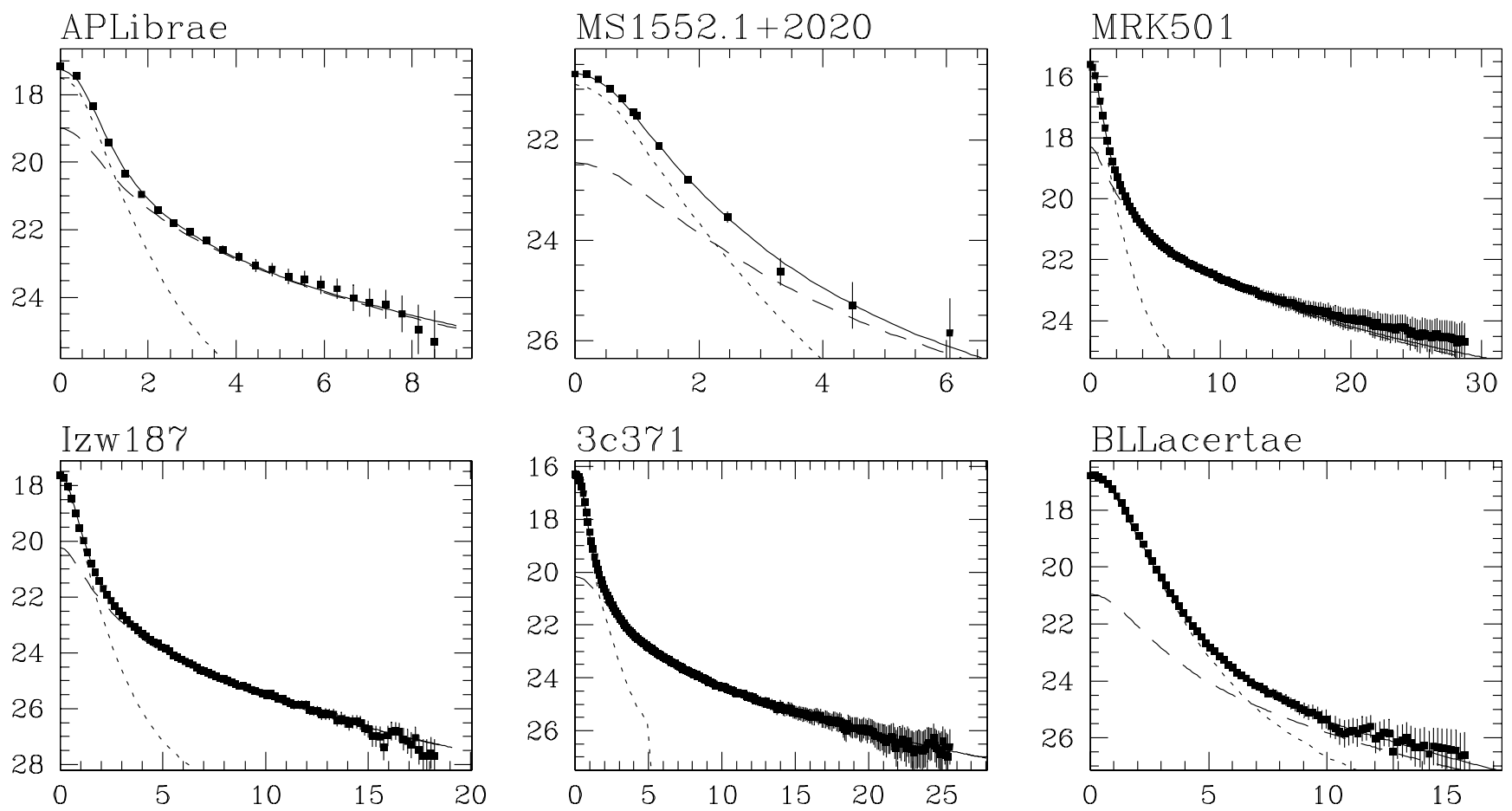

Fig. 9. continued.
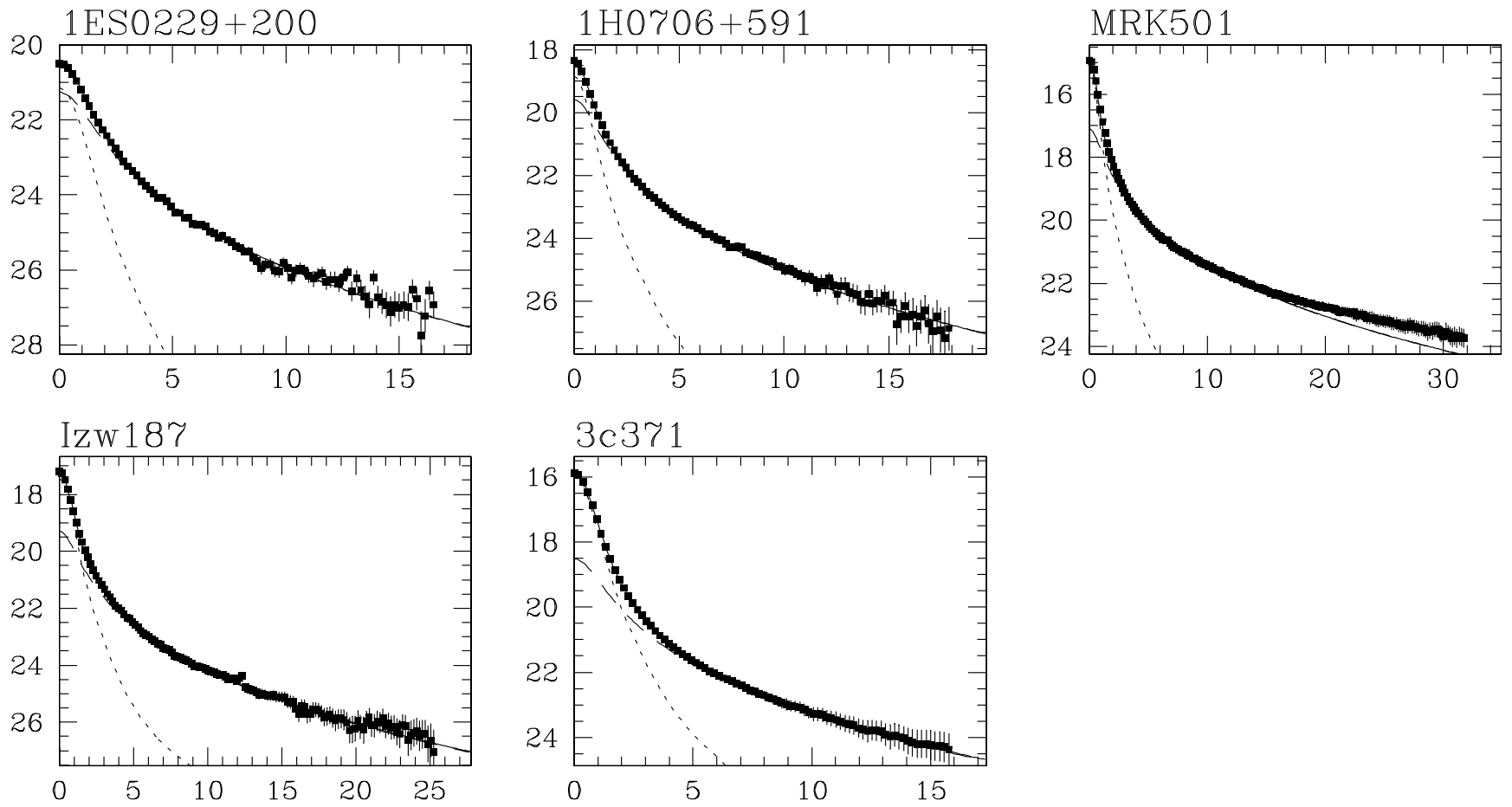

Fig. 10. The observed $V$-band radial luminosity profiles and model profiles. For explanation, see the caption of Fig. 8 . 